\title{
Probing the lower ionospheric perturbations associated with earthquakes by means of subionospheric VLF/LF propagation*
}

\author{
Masashi Hayakawa ${ }^{1,2, *}$ \\ ${ }^{1}$ Advanced Wireless Communications Research Center and Research Station on Seismo Electromagnetics, \\ The University of Electro-Communications, 1-5-1 Chofugaoka, Chofu Tokyo 182-8585, Japan \\ ${ }^{2}$ Earthquake Analysis Laboratory, Information Systems Inc., Kita-Aoyama 2-12-42-R305, Minato-ku, \\ Tokyo 107-0061, Japan
}

\begin{abstract}
There have been reports for many years that the ionosphere is very sensitive to seismic effects, and the detection of ionospheric perturbations associated with earthquakes (EQs) attracts a lot of attention as a very promising candidate for short-term EQ prediction. In this review we present a possible use of VLF/LF (very low frequency $(3-30 \mathrm{kHz}) /$ low frequency $(30-300 \mathrm{kHz})$ ) radio sounding of seismo-ionospheric perturbations. In order to avoid the overlapping with my own previous reviews, we first show some pioneering results for the Kobe EQ and we try to present the latest results including the statistical evidence on the correlation between the VLF/LF propagation anomalies (ionospheric perturbations) and EQs (especially with large magnitude and with shallow depth), medium-distance (6-8 Mm) propagation anomalies, the fluctuation spectra of subionospheric VLF/LF data (the effect of atmospheric gravity waves, the effect of Earth's tides, etc.), and the mechanism of lithosphere-atmosphere-ionosphere coupling. Finally, we indicate the present situation of this kind of VLF/LF activities going on in different parts of the globe and we suggest the importance of international collaboration in this seismo-electromagnetic study.
\end{abstract}

Key words: Earth-ionosphere waveguide; subionospheric VLF/LF propagation; seismo-ionospheric perturbation; earthquake; lithosphere-atmosphere-ionosphere coupling

CLC number: P352.3, P315.72 Document code: A

\section{Introduction}

One of most serious natural disasters is associated with earthquakes (EQs), and the media news for the latest, huge EQs such as Japanese Niigata EQ, Indonesia Sumatra EQ, Haiti EQ, etc. have indicated the great devastation inflicted by EQs. In order to mitigate the EQ disaster, it would be immensely useful to forecast the approach of a large EQ on a timescale of hours, days and weeks. However, in spite of the essential importance

\footnotetext{
* Received 13 May 2011; accepted in revised form

24 July 2011; published 10 December 2011.

† Corresponding author. e-mail: hayakawa@whistler.ee.uec.ac.jp

(c) The Seismological Society of China and Springer-Verlag Berlin Heidelberg 2011
}

of this short-term EQ prediction, it has been far from realization. The situation for the short-term EQ prediction seems to have been drastically changed in Japan during the last ten years since the Kobe EQ in 1995, because the conventional EQ prediction based on the measurement of crustal movements, has been concluded to be not so useful for the short-term EQ prediction. Then, we have had a new wave of the measurements by means of electromagnetic effects, and we have accumulated a substantial number of evidences that electromagnetic phenomena do take place in a wide frequency range prior to an EQ (e.g., Hayakawa, 2007, 2009a, b, 2010a; Hayakawa and Molchanov, 2002, 2007; Molchanov and Hayakawa, 2008). While the seismic (mechanical) mea- 
surement offers the 0th-order (or macroscopic) information of an EQ only after its occurrence, the higher-order (microscopic) phenomena are precursory to an EQ and can only be tackled by electromagnetic effects. This is the reason why the electromagnetic effects attract a lot of attention as a promising candidate of short-term EQ prediction.

The electromagnetic method for EQ prediction can principally be classified into two categories: the first is the detection of radio emissions from the EQ hypocenter (or epicenter), and the second is to detect an indirect effect of EQs taking place in the atmosphere and ionosphere by means of the pre-existing radio transmitter signals (we call it "radio sounding"). As the result of research during the last ten years, it has been a consensus that the ionosphere is unexpectedly extremely sensitive to the seismic effect (e.g., Hayakawa, 2009b; Hayakawa and Molchanov, 2002), which can be extensively studied by means of subionospheric VLF/LF propagation.

This review starts with our historical event of the 1995 Kobe EQ, followed by recent findings by Japanese network observation including a statistical correlation of ionospheric perturbations with EQs, VLF/LF propagation anomalies for the medium-distance propagation, VLF/LF fluctuation spectra, the effect of Earth's tides, and a case study of the 2010 Haiti EQ. Finally, the generation mechanism of seismo-ionospheric perturbations is discussed.

\section{Usage of VLF/LF subionospher- ic propagation as a new method- ology}

A number of nations currently operate large VLF/LF transmitters primarily for navigation and communication with military submarines. To radiate electromagnetic waves efficiently, one needs an antenna with dimensions on the order of a wavelength of the radiation, which suggests that VLF/LF transmitter antennas must be very large, typically many hundreds of meters high (Watt, 1967).

Most of the energy radiated by such VLF/LF transmitters is trapped between the ground and the lower ionosphere, forming the Earth-ionosphere waveguide. Subionospheric VLF/LF signals reflect from the D-region of the ionosphere, probably the least studied region of the Earth's atmosphere (Budden, 1961; Wait, 1962; Al'pert, 1983). These altitudes $(\sim 70-90 \mathrm{~km})$ are too far for balloons and too low for satellites, making in-situ measurements extremely rare. The only possible method for probing this $\mathrm{D} / \mathrm{E}$ region is $\mathrm{VLF} / \mathrm{LF}$ subionospheric radio signals.

So that any variations in the ionospheric $\mathrm{D} / \mathrm{E}$ region plasma lead to changes in the conditions for VLF waves propagating subionospherically, and hence changes in the observed amplitude and phase of VLF/LF transmissions are due to different kinds of perturbation sources: (1) solar flares (e.g., Mitra, 1974), (2) geomagnetic storms (and the corresponding particle precipitation) (e.g., Kikuchi and Evans, 1983), (3) the direct effect of lightning (e.g., Rodger and McCormic, 2006) and so on. In addition to these solar-terrestrial effects we can suggest one more effect of EQs (or seismic activity) onto the lower ionosphere as a new science topic.

\section{$3 \mathrm{VLF} / \mathrm{LF}$ probing of seismo- ionospheric perturbations}

\subsection{History of VLF / LF subionospheric method}

The most convincing evidence on the seismoionospheric perturbations with VLF sounding was obtained by Hayakawa et al. (1996b) for the famous Kobe EQ on 17 January, 1995. Some important peculiarities in their paper are summarized as follows: (1) the propagation distance from Tsushima Omega (geographic coordinates $34.37^{\circ} \mathrm{N}, 129.27^{\circ} \mathrm{E}$ ) to Inubo observatory $\left(35.42^{\circ} \mathrm{N}, 140.52^{\circ} \mathrm{E}\right)$ is relatively short at VLF $(\sim 1 \mathrm{Mm}$ (1 $000 \mathrm{~km})$ ), as compared with 5-9 Mm used in previous Russian papers (Gokhberg et al., 1989; Gufeld et al., 1992), and (2) they found that the nighttime fluctuation method as used before, was not so effective for the short-propagation path, so they developed another way of analysis so-called the terminator-time (TT) method. The TT is defined as the time when the diurnal phase (or amplitude) variation exhibits a minimum around sunrise and sunset (which we call morning $\left(t_{\mathrm{m}}\right)$ and evening $\left(t_{\mathrm{e}}\right)$ TTs). We found a significant shift in TTs before the EQ, that is, $t_{\mathrm{m}}$ shifts to early hours and $t_{\mathrm{e}}$ to later hours. This effect is also confirmed by analysis over a much longer data length $( \pm 4$ months; over a total of eight months). See the details in Hayakawa (2007, 2009a, 2010a) and Hayakawa et al. (1996b).

A later extensive study by Molchanov and Hayakawa (1998) was based on the much more events during 13 years (11 events with magnitude greater than 6.0 and within the first Fresnel zone) for the same propagation path from the Omega, Tsushima to Inubo, and 
they came to the following conclusion.

1) As for shallow (depth smaller than $30 \mathrm{~km}$ ) EQs, four EQs from five exhibited the same TT anomaly as for the Kobe EQ with the same $2 \sigma$ ( $\sigma$ presents standard deviation) criterion.

2) When the depth of EQs is in a medium range of 30-100 km, there were observed two events. One event exhibited the same TT anomaly, while another indicated a different type of anomaly.

3) Deep (depth larger than $100 \mathrm{~km}$ ) EQs (four events) did not accompany any anomaly. Two of them had an extremely large magnitude (greater than 7.0), but had no propagation anomaly.

This summary might indicate a relatively high probability of the propagation anomaly (in the form of TT anomaly) on the order of $70 \%-80 \%$ for larger EQs (magnitude greater than 6.0) located relatively close to the great-circle path (e.g., first Fresnel zone).

Another important finding is that when we have the propagation anomaly (ionospheric perturbations) a harmonic analysis on the data of the TTs exhibits an enhanced modulation with periodicities of 5 days or 9-11 days (these periods are those of planetary waves). This implies that atmospheric oscillations with those periodicities may play an important role in the coupling from the lithosphere to the ionosphere. Recently we proposed atmospheric gravity waves (AGWs) as the carrier because of their stronger tendency of upward propagation in the lithosphere-ionosphere coupling, with the planetary wave as the modulating signal (Hayakawa, 2009a). Based on the study of fluctuation spectra of our observational data (on amplitude and phase), we found an enhanced occurrence of fluctuation power in the frequency range (10 min to $2 \mathrm{~h}$ ) of AGWs, probably associated with EQs (Molchanow et al., 2001). These findings may provide a fundamental basis for the study of lithosphere-atmosphere-ionosphere coupling as will be discussed later.

Hayakawa et al. (1996b) and Molchanov et al. (1998) suggested to explain the change in the lower ionosphere by means of the full-wave theory of subionospheric VLF propagation over a short distance $(\sim 1 \mathrm{Mm})$ for which there exist several modes of propagation (i.e. $\mathrm{TT}$ is the consequence of wave interference of those modes). On the basis of the comparison of theoretical estimations with the experimental data, we concluded that the lower ionosphere might have been lowered by a few kilometers. Here we present a comprehensive view on the importance of TT shift in the subionospheric $\mathrm{VLF} / \mathrm{LF}$ diurnal variation and its use in inferring the lower ionopsheric changes associated with EQs. Unlike the works mentioned above based on the full-wave computations (Hayakawa et al., 1996b), Yoshida et al. (2008) made full use of wave hop method (theory) to interpret the TT changes in terms of the wave interference between the ground and sky waves, and indicated how to estimate the change (normally decrease) in ionospheric height by means of the observed shift of TTs.

\subsection{Japanese VLF/LF network}

In response to the above-mentioned significant results (especially the result for Kobe EQ), the Japanese government conducted the integrated EQ frontier project, and the former NASDA (National Space Development Agency of Japan) conducted the so-called "Earthquake Remote Sensing Frontier Project" (for which the author was the principal investigator) during the period of 1997 through 2001 (five years project) (Hayakawa, 2004; Hayakawa et al., 2004a, b). As the name of this project suggests, the main concern of the former NASDA was the remote sensing of different regions including the lithosphere, atmosphere, and upper atmosphere. As already summarized in our previous papers (Hayakawa, 2004), we have analyzed the seismic effects not only in the lithosphere, but also in the upper atmosphere. The finding of seismo-ionospheric perturbations for the Kobe EQ was an extremely big surprise even for us, that is, the fact that the upper atmosphere or the ionosphere is so sensitive to the pre-seismic activity. Another important finding was the discovery of ULF (ultra-low-frequency, frequency less than $5 \mathrm{~Hz}$ ) electromagnetic emissions. That is, we have used a new analysis method called polarization method (the ratio of vertical to horizontal magnetic field components), which enabled us to find a significant effect of seismogenic ULF emissions (an extremely good way to identify the EQ ULF noise from other dominant noises) (Hayakawa et al., 2007a). The acoustic emission was also found to be a promising candidate for short-term EQ prediction (see Hayakawa, 2004). A further effect in the lithosphere is our finding on the surface temperature anomaly associated with EQs, which are detected by using the thermal images from satellites (Hayakawa, 2004; Tronin et al., 2002).

In addition to these effects in the lithosphere, we have tried to find out any EQ signature in the atmosphere and ionosphere. The atmospheric perturbation is also identified by means of over-horizon VHF transmitter signal (this is a kind of integrated measurement) (Fukumoto et al., 2001; Fujiwara et al., 2004; Hayakawa, 2009c; Hayakawa et al., 2007b), and the gen- 
eration mechanism of these atmospheric perturbations has recently been proposed by Hayakawa et al. (2007b) based on the geochemical effects prior to an EQ.

In the project of the former NASDA's frontier project, we paid our greatest attention to subionospheric $\mathrm{VLF} / \mathrm{LF}$ propagation aimed at the short-term EQ prediction. Figure 1 is the Japanese VLF/LF network established within the framework of the frontier project and is still working actively. Figure 1 shows seven observing stations, i.e., Moshiri (Hokkaido) (abbreviated as MSR), Chofu (Tokyo) (CHF), Tateyama (Chiba) (TYM), Shimizu (Shizuoka) (SMZ), Kasugai (Nagoya) (KSG), Maizuru (Kyoto) (MZR) and Kochi (KCH), but the three observatories (TYM, SMZ, MZR) were ceased some time ago. An additional new observatory is established recently; Tsuyama in Okayama prefecture (TYM, accidental coincidence of abbreviaion with the former TYM). We observe signals from several transmitters simultaneously at each station, unlike the early VLF receiving system. The VLF/LF transmitters we observe now, are (1) JJY (40 kHz, Fukushima), (2) JJI



Figure 1 Map of Japanese VLF/LF network. It consists of several receiving stations (Moshiri (MSR), Chofu (CHF), Tateyama, Chiba (TYM), Shimizu (SMZ), Kasugai, Nagoya (KSG), Maizuru (MZR) and Kochi (KCH)). At each station we receive simultaneously several VLF/LF transmitters including two Japanese, JJY and JJI and three foreign ones (NWC, NPM, NLK). The propagation paths for those transmitters are shown only at MSR as an example.
(22.2 kHz, Ebino, Kyushu), (3) NWC (19.8 kHz, Australia), (4) NPM (21.4 kHz, Hawaii) and (5) NLK (24.8 $\mathrm{kHz}$, America). By using the combination of a number of observing stations and a large number of VLF/LF transmitters received, we will be able to locate the ionospheric perturbation with the accuracy of about $100 \mathrm{~km}$.

We make some comments on our AbsPAL (Absolute Phase and Amplitude Logger) system. Our $\mathrm{VLF} / \mathrm{LF}$ receiver is designed to measure very slow and small changes in amplitude and phase. The magnitude of slow phase and amplitude perturbations claimed for EQ precursors are significant, so it should be detectable by our system if they exist. The block diagram of this AbsPAL is given in Figure 2 (R. L. Dowden, private communication). The wideband signal received by a vertical monopole antenna is mixed with the local oscillator with the frequency of any VLF/LF transmitter in order to measure the amplitude and phase of the transmitter signal (see Figure 2). How to create the signal for the local oscillator is illustrated in Figure 3. In order to

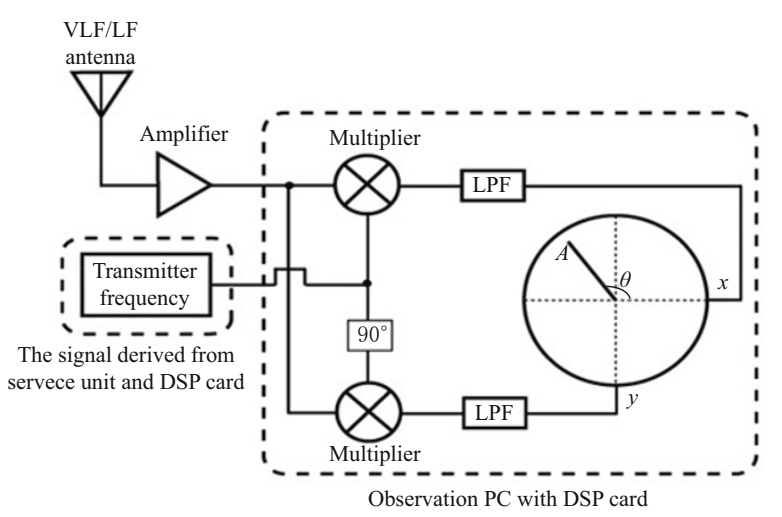

Figure 2 Principle of measuring the amplitude and phase of VLF/ LF transmitter signals in our VLF/LF receiver system.

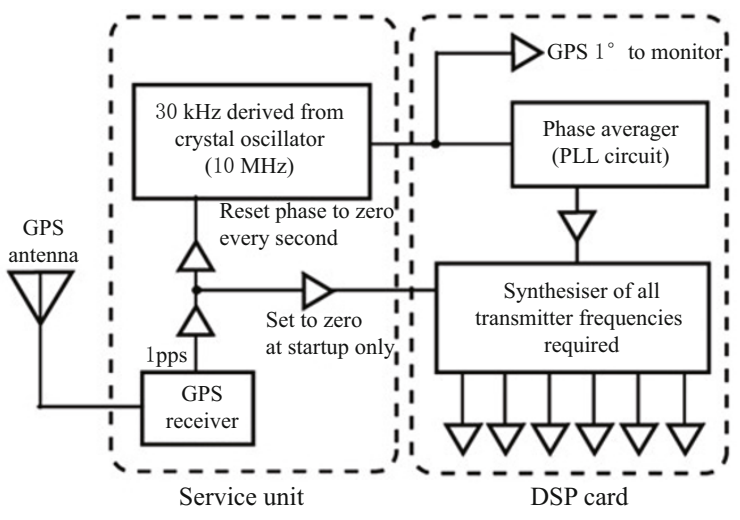

Figure 3 Block diagram of generating the signal for the local oscillator with the GPS signal and the system of synthesis of transmitter frequencies. 
choose several transmitter signals (maximum 6), we make, in the service unit, a reference signal with a frequency $30 \mathrm{kHz}$ by means of a crystal oscillator of 10 $\mathrm{MHz}$ and the associated signal synthesis circuit. This $30 \mathrm{kHz}$ signal is fed to a phase averager based on the PLL (phase lock loop) circuit in the DSP card as in Figure 3. Together with this signal and the GPS 1 pps signal, we will be able to synthesize all necessary transmitter frequencies in the DSP card. Figure 4 illustrates the overall observation system at any station. The data observed there are stored in the observation PC, and they are transmitted to the server PC in our laboratory in Chofu (CHF) via a telephone line or an internet link by means of the transmission PC.

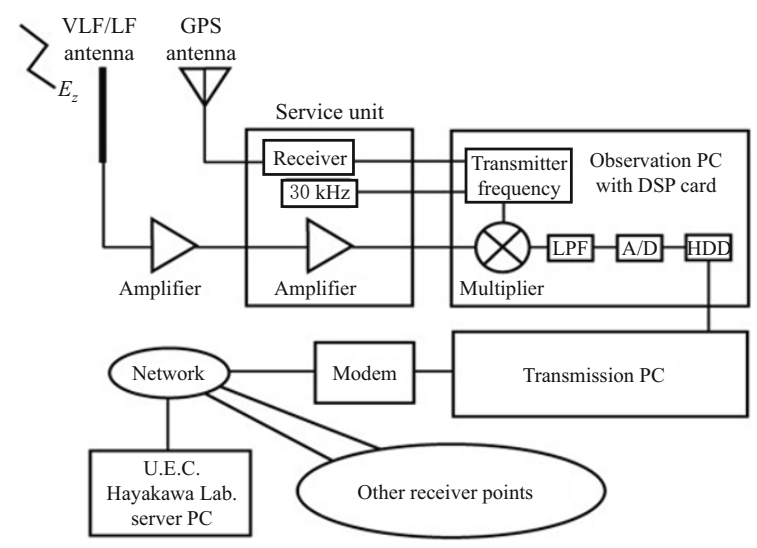

Figure 4 Overall system of observation and data transmission at one of the VLF/LF receiving stations.

Our VLF/LF system is deployed in different counties as well in response to their requests. One of our $\mathrm{VLF} / \mathrm{LF}$ receivers is now working at Kamchatka in Russia with good results (Rozhnoi et al., 2004), and one was also installed in Taiwan of China (Hayakawa et al., 2010a). These stations, together with our Japanese dense network, are forming a global Asian-Pacific VLF/LF network.

\section{Recent findings from Japanese VLF/LF network}

We here present our latest results just published or to be published shortly.

\subsection{Statistical study on the correlation be- tween VLF/LF propagation anomalies} (ionospheric perturbations) and EQs

There have been published only very few papers reporting the statistical correlation between the VLF/LF propagation anomalies and EQs (Rozhnoi et al., 2004;
Maekawa et al., 2006; Kasahara et al., 2008). Rozhnoi et al. (2004) analyzed the data observed in PetropavloskKamchatka (KCK) in Russia, where they received the LF signals from a Japanese transmitter JJY in Figure 1. The length of this wave-path is about $2300 \mathrm{~km}$, and the period of analysis was 2 years. Unlike the TT method as used initially for the Kobe EQ, Rozhnoi et al. (2004) used the so-called nighttime fluctuation method, in which they pay attention only to the nighttime data. As the result of analysis, they found that the sensitivity of LF signals (both amplitude and/phase and their dispersions) to seismic activity becomes apparent mainly for large EQs with magnitude greater than 5.5. And, the anomaly takes place a few days before and several days after an EQ.

Then, Maekawa et al. (2006) have attempted the similar statistical correlation between the VLF/LF propagation anomalies and EQs on the basis of observation during a much longer period of six years. But again they used only a particular wave-path from the same JJY transmitter and a receiver at Kochi $(\mathrm{KCH})$ in Figure 1. They used again the same nighttime fluctuation method (Rozhnoi et al., 2004; Shvets et al., 2002, 2004), which will be explained later. They used a residual signal of amplitude $(\mathrm{d} A(t))$ as the difference between the observed signal intensity and the average over several days preceding or following the current day. Only local nighttime period is adopted (e.g., UT $=10 \mathrm{~h}$ to 20 $\mathrm{h}$ or $\mathrm{LT}=19 \mathrm{~h}$ to $5 \mathrm{~h}$ ). As the result of their analysis, the trend defined as the nighttime average amplitude is found to show a significant decrease exceeding the $2 \sigma$ ( $\sigma$ represents standard deviation) a few days before the EQ when EQ magnitude is greater than 6.0.

Here in order to elaborate the above few studies, we present our latest results on the further statistical study on the correlation between VLF/LF propagation anomalies and EQs on the basis of a much longer period of analysis and more propagation paths (Hayakawa et al., 2010b). Many propagation paths will be available by combination of selecting different transmitters and receivers as in Figure 1. However, after checking the quality of the data for all possible propagation paths, we have chosen only the following wave paths with sufficient data quality for analysis, whose wave sensitive areas are illustrated as elliptic regions in Figure 5.
1. JJY-KCH
2. JJY-MSR
3. JJY-KCK
4. JJI-TYM
5. JJI-MSR 


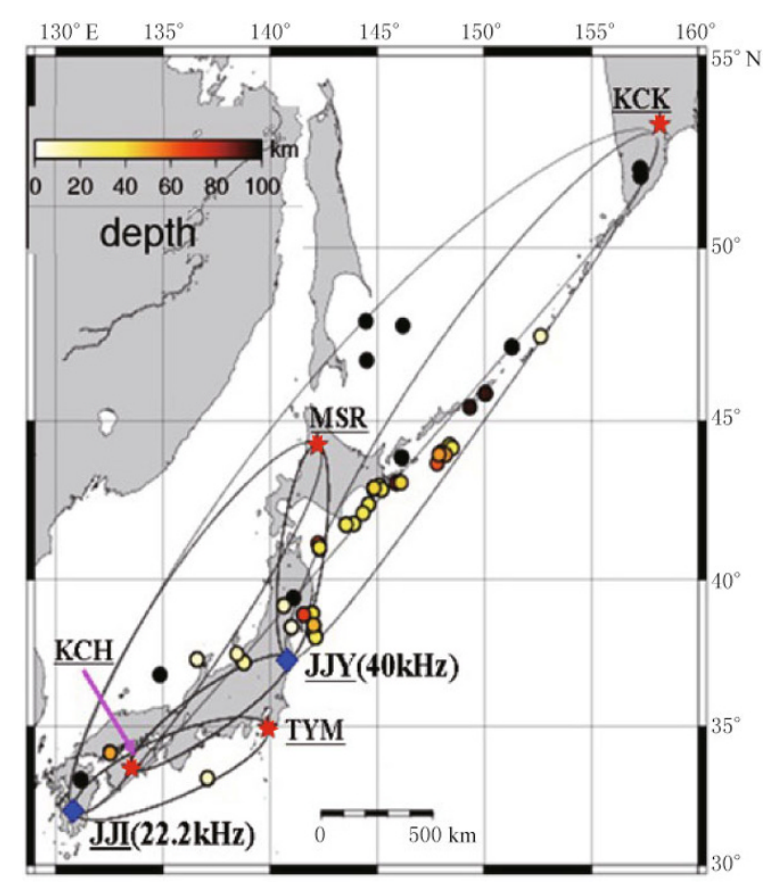

Figure 5 The relative locations of two Japanese transmitters. Call signs, JJY (40 kHz, Fukushima) and JJI (22.2 kHz, Ebino, Kyushu) are indicated by blue diamonds and four observing stations (Moshiri (MSR), Kamchatka $(\mathrm{KCK})$, Kochi $(\mathrm{KCH})$ and Tateyama, Chiba (TYM)) in red stars. EQs treated are also plotted, with their color indicating the EQ depth. Wave sensitive areas (defined by the fifth Fresnel zone) are also plotted for all propagation paths.

\section{JJI-KCK}

The wave sensitive area for each propagation path is defined by the fifth Fresnel zone of the great-circle path as adopted in the previous works (Maekawa et al., 2006; Kasahara et al., 2008). All of the data received at all the receiving stations are sampled every $120 \mathrm{~s}(2$ min). Only the amplitude data are analyzed here, because the phase data are sometimes not good enough for further analysis.

The period of analysis is considerably extended as compared with the longest period of four years in Kasahara et al. (2008). That is, we have used the data over total seven years from January 1, 2001 to December 31, 2007 (to be more exact, up to October 31, 2007 only for the paths JJY-KCK and JJI-KCK). It seems that this would be the longest analysis period so far in the field of seismo-electromagnetic studies.

Based on the previous statistical studies (Rozhnoi et al., 2004; Maekawa et al., 2006; Kasahara et al., 2008), the magnitude of 5.5 is found to be just at the border to obtain any significant correlation with $2 \sigma$ criterion between the VLF/LF propagation anomalies and
EQs, so that we choose the magnitude of 6.0 here as a rather severe criterion of selecting EQs. By imposing this condition, we have found 37 EQs taking place within the wave sensitive areas defined by the fifth Fresnel zones of the great-circle paths.

For our analysis we divide the EQ depth into two regions - shallower or deeper than $40 \mathrm{~km}$ in order to find the dependence on EQ depth. As seen from the configuration of the propagation paths in Figure 5, we can imagine that some EQs are common on a few propagation paths. In the case of shallow EQs (depth $<40$ $\mathrm{km}$ ), three EQs are common for three paths and 13 EQs are common for two paths. Similarly, for deep EQ (depth $\geq 40 \mathrm{~km}$ ), four EQs are seen for three paths and 16 EQs are common on two paths. Therefore, the total number of propagation paths which cover the EQs with depth smaller than $40 \mathrm{~km}$ is 35 ; while the corresponding number is 38 for EQs with depth larger than $40 \mathrm{~km}$. This is summarized in Table 1. We treat the data for each propagation path as independent events, so that the total number of events is 73. This independent treatment seems to be validated by our previous work. Yamauchi et al. (2007) have examined a few propagation paths for a particular and large EQ named the 2004 Mid-Niigata EQ by means of the TT method, and then they have found that the anomaly in TT does not happen always on the same day. This might suggest that the seismoionospheric perturbation is very inhomogeneous both in space and in time, leading to the effect of very dissimilar variations on different propagation paths even for the same EQ. Here we use the nighttime fluctuation method, and we describe briefly the way of analysis. The reason for using only the nighttime data is that the daytime amplitude exhibits too small changes to analyze. Figure 6 illustrates the diurnal variation (amplitude) for one particular path on a particular day in UT (given in red), in which you can identify clearly the evening and morning TTs (both defined in LT). The local nighttime period is indicated in shadow. We define the difference (or residue) as follows:

Table 1 EQs treated

\begin{tabular}{ccc}
\hline Path & EQs with depth $<40 \mathrm{~km}$ & EQs with depth $\geq 40 \mathrm{~km}$ \\
\hline JJY-MSR & 6 & 4 \\
JJY-KCK & 15 & 15 \\
JJI-TYM & 1 & 1 \\
JJI-MSR & 1 & 3 \\
JJI-KCK & 10 & 15 \\
JJY-KCH & 2 & 0 \\
Total & 35 & 38 \\
\hline
\end{tabular}



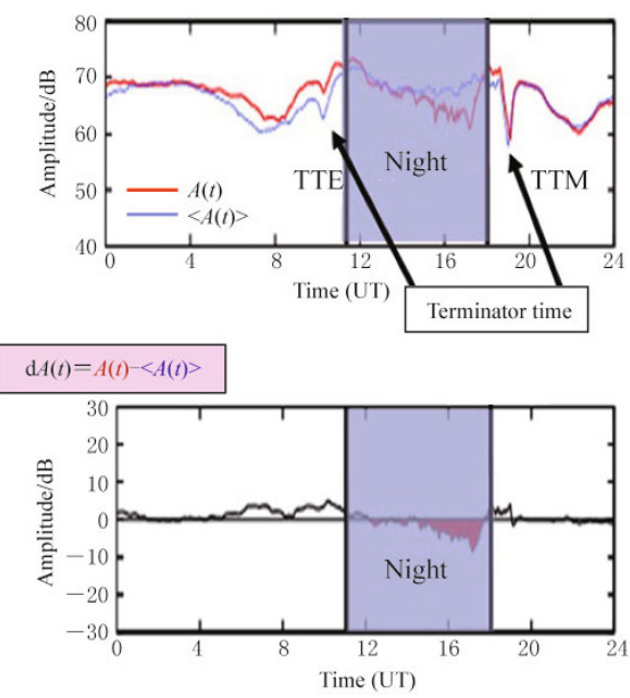

Figure 6 Explanation of the analysis of VLF/LF amplitude data. Top panel indicates the diurnal variation (in red) for a particular time $t$ on a particular day $(A(t))$ and the corresponding variation (in blue) averaged over \pm 15 days of the day $(<A(t)>)$. The bottom refers to the difference of $\mathrm{d} A(t)=A(t)-<A(t)>$ as a residue.

$$
\mathrm{d} A(t)=A(t)-<A(t)>,
$$

where $A(t)$ is the amplitude at a time $t$ on a current day and $\langle A(t)\rangle$ is the running average at the same time $t$ over \pm 15 days around the relevant day (before and after the relevant day). The residue is plotted in the lower part of Figure 6, and by using this kind of figure we estimate the following three physical quantities of amplitude (Rozhnoi et al., 2004; Maekawa et al., 2006): (1) trend (as the average of nighttime amplitude)(in $\mathrm{dB}$ ), $(2)$ dispersion $(D)$ (in the following we use its square root, i.e., standard deviation, but we use the terminology of dispersion in order to avoid the confusion that the standard deviation for each quantity is used very often in this paper), (3) nighttime fluctuation (NF).

$$
\begin{aligned}
& \text { trend }=\frac{\int_{N_{\mathrm{s}}}^{N_{\mathrm{e}}} \mathrm{d} A(t) \mathrm{d} t}{N_{\mathrm{e}}-N_{\mathrm{s}}}, \\
& \mathrm{NF}=\int_{N_{\mathrm{s}}}^{N_{\mathrm{e}}}(\mathrm{d} A(t))^{2} \mathrm{~d} t,
\end{aligned}
$$

where $N_{\mathrm{s}}$ and $N_{\mathrm{e}}$ are the times of starting and ending the night-time in our analysis (we have decided $N_{\mathrm{s}}=11 \mathrm{~h} \mathrm{UT}$ and $N_{\mathrm{e}}=18 \mathrm{~h}$ UT after looking at the diurnal variations) and we know that $\mathrm{d} A(t)<0$ is essential for our seismogenic effects (Maekawa et al., 2006; Kasahara et al., 2008) so that only $\mathrm{d} A(t)<0$ is used for the estimation of NF. Maekawa et al. (2006) and Kasahara et al. (2008) have indicated that any seismogenic effect is characterized by the almost simultaneous significant decrease in trend and significant increases in dispersion and NF.

Next we have to mention how to treat the data on different propagation paths, because the variability in VLF/LF amplitude data is very different from one path to another. So that, it is highly required to homogeneously treat the VLF/LF data when we analyze different propagation paths. We have proposed so-called "standardization" in the following way. That is, when taking one particular path, we deal with three physical quantities of amplitude, trend, $D$ and NF and we estimate the following normalized trend (trend ${ }^{*}$ ), normalized $D\left(D^{*}\right)$, and normalized $\mathrm{NF}\left(\mathrm{NF}^{*}\right)$. When taking an EQ with a particular date, we estimate the trend on this day and then calculate the average $<$ trend $>$ over \pm 15 days around this date. Then, the normalized trend $\left(\right.$ trend $\left.^{*}\right)$ is defined as (trend $-<$ trend $\left.>\right) / \sigma_{\mathrm{T}}\left(\sigma_{\mathrm{T}}\right.$ is standard deviation over \pm 15 days around the current date). The same principle is applied to other quantities in order to obtain the normalized $D\left(D^{*}\right)$ and normalized $\mathrm{NF}\left(\mathrm{NF}^{*}\right)$.

By using these normalized (or standardized) trend, $D$ and NF, we make full use of a superimposed epoch analysis (e.g., Rozhnoi et al., 2004; Maekawa et al., 2006), which is of extreme importance in enhancing the signal to noise ratio by stacking the data on EQ day as a reference day. Although we have chosen the EQs with magnitude greater than 6.0, but we pay more attention to the effect of EQ depth here because this point is poorly studied even though Maekawa et al. (2006) have suggested this point qualitatively.

Figures $7 \mathrm{a}, \mathrm{b}$ and $\mathrm{c}$ are the final trend ${ }^{*}, D^{*}$, and $\mathrm{NF}^{*}$ on the basis of superimposed epoch analysis. We can deduce from these figures the following summary.

1) The trend (or trend* in Figure 7a) is found to show a significant decrease (exceeding the $2 \sigma_{\mathrm{T}}$ criterion) before the shallow EQ (with depth $<40 \mathrm{~km}$ ) (in red). This anomaly takes place five days before the EQ as a conspicuous peak. When the EQ depth becomes larger (like more than $40 \mathrm{~km}$ in Figure $7 \mathrm{a}$ ), the similar tendency is likely to be observed in blue line in Figure $7 \mathrm{a}$ in such a way that the trend approaches the $2 \sigma_{\mathrm{T}}$ criterion 12 days before the EQ (but not exceeding the $2 \sigma_{\mathrm{T}}$ criterion).

2) Next the nighttime dispersion $\left(D^{*}\right)$ for EQ depths smaller than $40 \mathrm{~km}$ (in red) in Figure $7 \mathrm{~b}$ is found to exhibit a significant increase three days before the EQ (exceeding the $2 \sigma_{D}$ criterion and even approaching 

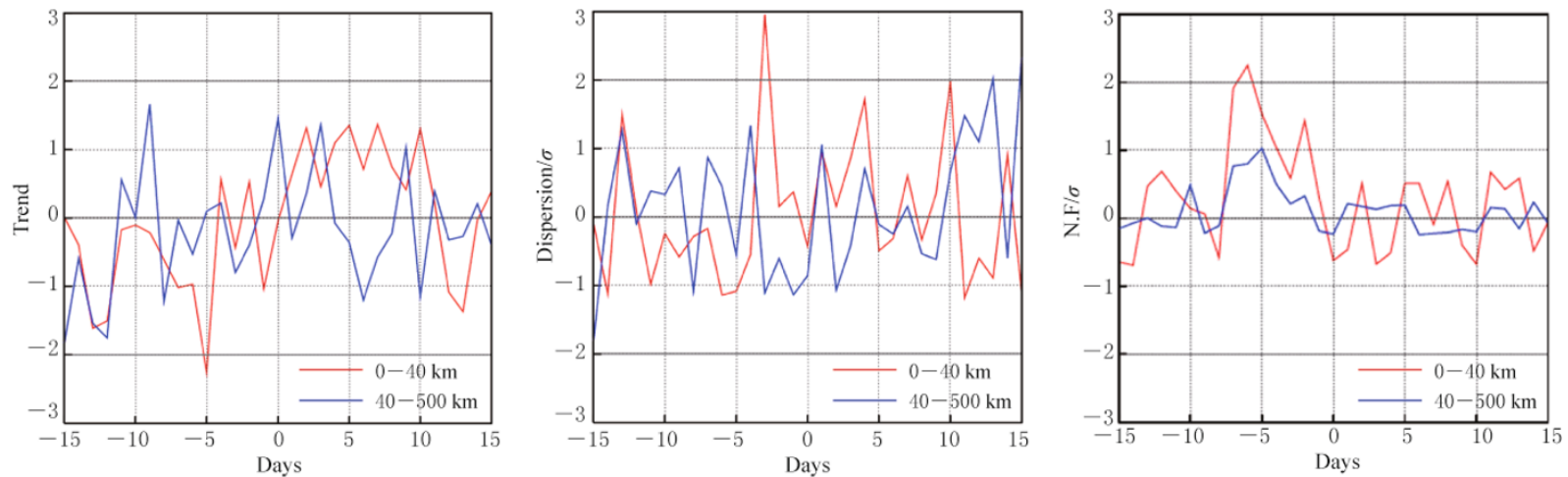

Figure 7 Superimposed epoch analysis for the normalized trend (trend*) (a), the normalized dispersion $(D)$ (dispersion*) (b), and the normalized NF ( $\left.\mathrm{NF}^{*}\right)$ (c). The red line refers to shallow EQs (depth $\left.<40 \mathrm{~km}\right)$, and the blue line refers to EQs with depth larger than $40 \mathrm{~km}$. The abscissa indicates the day with respect to the EQ day (0), that is -(minus) means the day before the EQ and +(plus), the day after the EQ. After Hayakawa et al. (2010b).

$3 \sigma_{D}$ level). However, when the EQ depth becomes larger than $40 \mathrm{~km}$ (in blue line in Figure $7 \mathrm{~b}$ ), there is no clear precursory effort before such a deep EQ.

3) Figure $7 \mathrm{c}$ concerning the NF is found to indicate significant enhancements only before the EQ (5-6 days before the $\mathrm{EQ}$ ) with exceeding the $2 \sigma_{\mathrm{NF}}$ criterion. No such enhancements in NF are detected for EQs with depth larger than $40 \mathrm{~km}$.

Then we describe some other possible effects on VLF/LF perturbations as mentioned before. The most conventional solar-flare effects are too short in duration (less than 10 minutes), so that there is no problem to identify them in the VLF/LF data. The next extraterrestrial effect such as gamma-ray stars (e.g., Tanaka et al., 2008) is seen to be similar in duration to the solarflare phenomenon. The last solar-terrestrial effect might be geomagnetic storms, which take place during a few days one or two days after the onset of a geomagnetic storm. In this sense, this effect might be the most serious problem to our seismo-ionospheric perturbation, which will be discussed later. Finally, there exists an atmospheric effect such as the impact of a lightning discharge onto the ionosphere due to direct heating/ionization, which is short in duration (as early/fast trimpis) (Dowden et al., 1994; Inan et al., 1996; Rodger, 1999), so that this effect is easily distinguished from the seismoionospheric effect. The lightning-induced particle precipitation due to wave-particle interaction in the magnetosphere (so-called "classical" trimpis) is again short in duration (on the order of a few min (e.g., Rodger, 1999)), and then there is no problem for us to identify this effect. All of these effects mentioned above can be easily detected because we know the exact times of these phenomena. While, seismo-ionospheric perturbation is the only exception because we do not know when it would happen.

The most confounded effect might be the geomagnetic storms (e.g., Liu et al., 2006). When obtaining Figure 7 we have paid no attention to the geomagnetic activity at all. Here we comment on the effect of geomagnetic storms on the ionospheric perturbation because Kleimenova et al. (2004) have presented an example of the effect of geomagnetic storms on subionospheric VLF propagation. As already found from Figure 7 , the seismo-ionospheric disturbances are found to take place before an EQ (on the order from day 0 to about 10 days before the EQ), so that we have examined the geomagnetic activity and geomagnetic storm for all EQs treated. We have examined the geomagnetic activity for all EQs, that is, we have estimated the mean $3 \mathrm{~h}-K_{\mathrm{p}}$ index for 10 days prior to each EQ. Then, it is found that the average $3 \mathrm{~h}-K_{\mathrm{p}}$ index during these 10 days is less than 2.0 for $32 \%$ of EQs, $2.0-3.0$ for $46 \%$ of EQs, 3.0-4.0 for 19\% of EQs and 4.0-5.0 for 3\% of EQs. So, we can say that the geomagnetic activity for all EQs was accidentally not so active. However, it is better to analyze the geomagnetic storms which are a non-stationary activity and which would influence the lower ionosphere. We have checked whether there are geomagnetic storms during 10 days prior to all EQs. As the result, one EQ accompanied two relatively large geomagnetic storms (|Dst $\mid=359$ and $460 \mathrm{nT}$ ), and there were only several EQs with small $\mid$ Dst $\mid(\mid$ Dst $\mid<200 \mathrm{nT})$. So it might be possible that only one particular EQ 
might be disturbed by the geomagnetic activity, but it is reasonable to think that all of the ionospheric perturbations are the consequence of EQs (those results in Figure 7).

\subsection{VLF/LF anomaly on medium-distance propagation paths}

A majority of our previous papers are based on the data over relatively short distance (distance is from 1 $\mathrm{Mm}$ to a few $\mathrm{Mm}$ ) propagation. We have presented a very convincing evidence on ionospheric perturbations associated with the Kobe EQ (Hayakawa et al., 1996) in subsection 3.1, and this finding was based on the daily trace of the TTs by using the Omega VLF transmitter located at Tsushima and by receiving this signal at Inubo. The shift of this TT effect has been investigated by Clilverd et al. (1999) for an extremely long (more than $10 \mathrm{Mm}$ ) NS (north-south) propagation path, and concluded that there existed no seismo-ionospheric perturbation and that the TT method is not effective in finding out seismo-ionospheric perturbations. It is rather easy for us to consider that it is reasonable for them to have detected no TT anomaly on their long propagation path for the following reasons. The first reason is that the perturbed region is too small as compared with the whole propagation path to expect any propagation anomaly. The second reason is that their NS propagation itself is generally not so suitable to detect any TT effect (Maekawa and Hayakawa, 2006).

In our recent paper by Kasahara et al. (2010) we pay attention to a medium distance (about 6-8 Mm) propagation path and we focus on several EQs for this distance propagation path. Unlike the above-mentioned TT method to identify seismo-ionospheric effect, we use here the nighttime fluctuation method (Rozhnoi et al., 2004; Hayakawa et al., 2010b; Maekawa and Hayakawa, 2006; Horie et al., 2007) as in the previous subsection in order to examine whether we will be able to detect any seismo-ionospheric perturbation for the mediumdistance propagation and to study the dependence of seismo-ionospheric disturbances on the distance of an EQ epicenter from the great-circle path, the effect of EQ depth, etc.

Here we focus on the propagation paths associated with the Australian NWC VLF transmitter located at northwest cape of Australia. Three observing (or receiving) stations from the Japanese VLF/LF network (Hayakawa, 2007) have been used, i.e., Moshiri (MSR), Kochi $(\mathrm{KCH})$, and Chofu (CHF) as shown in Figure 1. The distance from the transmitter (NWC) is $6.5 \mathrm{Mm}$ for $\mathrm{KCH}, 6.9 \mathrm{Mm}$ for $\mathrm{CHF}$ and $7.8 \mathrm{Mm}$ for MSR, respectively.

The period of data analysis is approximately six months from August 12008 to January 10 2009. During this time period we had relatively a large number of EQs in the Asian countries. Five large EQs took place, and we list them in Table 2. The positions of epicenters of those EQs are plotted in Figure 8, together with the information about EQ depth (in color). The corresponding wave sensitive area defined by the fifth Fresnel zone is also plotted in Figure 8 for each propagation path.

Table 2 EQs treated

\begin{tabular}{|c|c|c|c|c|c|c|}
\hline \multicolumn{2}{|c|}{ Origin time (UTC) } & \multicolumn{2}{|c|}{ Geographic coordinate } & \multirow{2}{*}{ Magnitude } & \multirow{2}{*}{ Depth/km } & \multirow{2}{*}{ Location } \\
\hline a-mo-d & $\mathrm{h}: \min : \mathrm{s}$ & Lat./ & Long./ & & & \\
\hline 2008-08-15 & $10: 25: 16$ & $12.90^{\circ} \mathrm{N}$ & $124.32^{\circ} \mathrm{E}$ & 6.0 & 10 & Philippines \\
\hline 2008-09-11 & 00:00:02 & $1.88^{\circ} \mathrm{N}$ & $127.36^{\circ} \mathrm{E}$ & 6.6 & 96 & Indonesia \\
\hline 2008-11-16 & $17: 02: 32$ & $1.27^{\circ} \mathrm{N}$ & $122.09^{\circ} \mathrm{E}$ & 7.3 & 30 & Indonesia \\
\hline 2008-12-06 & $10: 55: 26$ & $7.39^{\circ} \mathrm{S}$ & $124.73^{\circ} \mathrm{E}$ & 6.4 & 404 & East Timor \\
\hline 2009-01-03 & $22: 33: 40$ & $0.41^{\circ} \mathrm{S}$ & $132.89^{\circ} \mathrm{E}$ & 7.6 & 17 & Papua New Guinea \\
\hline
\end{tabular}

The nighttime fluctuation method is again adopted, so that we analyze (1) trend, (2) dispersion, (3) NF and (4) AGW (atmospheric gravity wave) modulation index. The first three physical and statistical quantities have been used extensively in 4.1 in order to find out the presence of seismo-ionospheric perturbations, but the last parameter of AGW modulation index is recently suggested to be analyzed (Muto et al., 2009b) and to be discussed later extensively in the next subsec- tion in order to confirm further the presence of seismoionospheric perturbations which seems to be closely related to their generation mechanism (or lithosphereatmosphere-ionosphere coupling).

Analysis results are presented for the three propagation paths, that is from NWC to $\mathrm{KCH}$, from NWC to CHF, from NWC to MSR. By taking Figure 9 as an example for the path from $\mathrm{NWC}$ to $\mathrm{KCH}$, we need to explain what are described in the figure. You notice four 


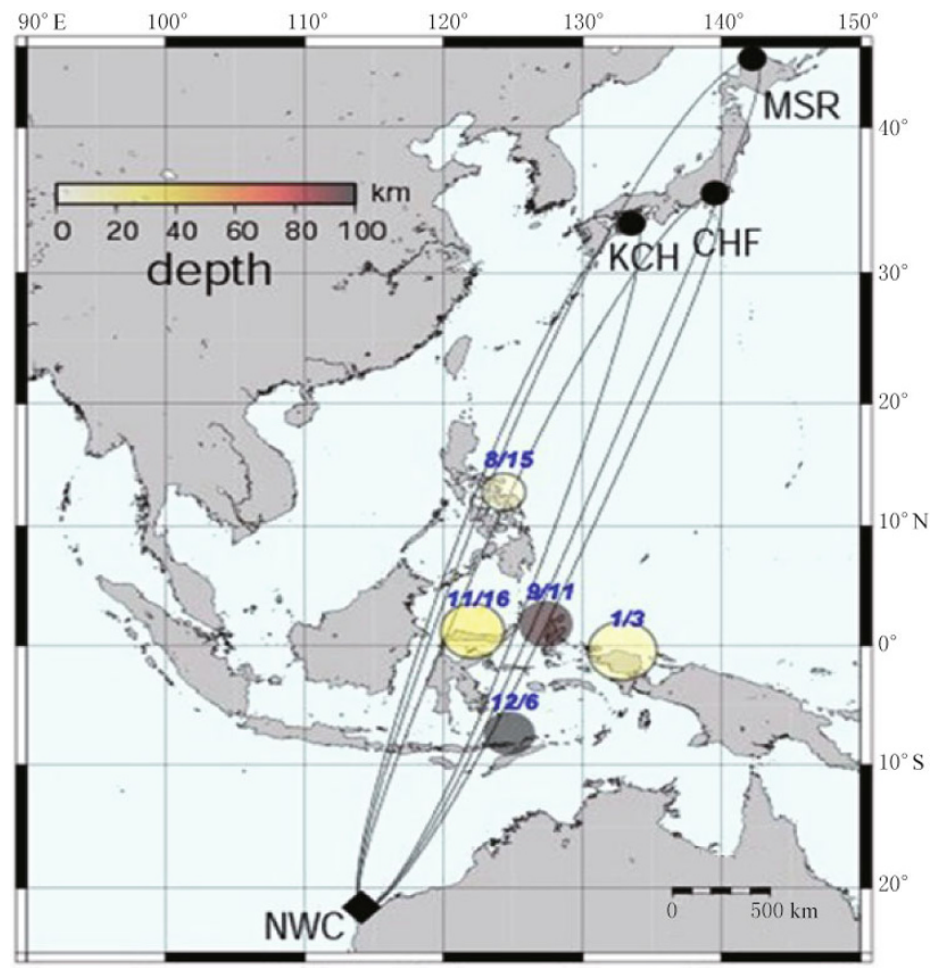

Figure 8 The relative locations of the transmitter, NWC, and three Japanese receiving stations, Moshiri (MSR), and Chofu (CHF) and Kochi $(\mathrm{KCH})$, together with the relevant wave sensitive area (defined by the fifth Fresnel zone). Also, the epicenters of five EQs are indicated, together with the information about the EQ depth (in color).

panels in the figure: from the top to the bottom, trend, dispersion, NF and AGW modulation index (this index will be explained later in subsection 4.3). The ordinate for each parameter is given in terms of its standard deviation $(\sigma)$, so that we normally pay attention to $\pm 2 \sigma$ as a criterion. The date is indicated on the bottom of each panel, starting from August 1, 2008 to the end of January, 2009. Five EQs in Table 2 are described in the top of the first panel (including the date, EQ magnitude and depth), in which the main EQ with the biggest magnitude is given in a yellow box. In addition to these five EQs, there are also indicated two significant EQs in the beginning and by the end of our analysis: August 4, 2008 and January 22, 2009 EQs. The times and magnitudes of these EQs are indicated by downward red bars on the top of each panel. The period in gray indicates the period of no observation due to different problems. Some values are indicated even for grey periods, but they are meaningless (should be blank).

As already known from the previous subsection, the trend tends to decrease down to $-2 \sigma$ level, while three other parameters tend to be enhanced over their corresponding $2 \sigma$ criteria. When these criteria are satis- fied, the period is likely to be seismogenic and it would be a precursory signature of an EQ. In the figure a red bar means that all of the four parameters satisfy the $2 \sigma$ criteria, a yellow bar means that three of the four parameters satisfy their $2 \sigma$ level, and a green bar indicates that two of the four parameters satisfy their $2 \sigma$ level. So we are ready to look at each carefully and try to understand whether there existed any precursory EQ signature or not.

We look at Figure 9 for the path from NWC-KCH. Though there are a lot of periods with data gaps (in gray), we will try to find out any propagation anomalies. Even though there are some data gaps before the first EQ on August 15, we could find out an anomaly about 10 days before the EQ (indicated by a yellow bar), which would appear to be a precursor to the EQ. You see a green box on the EQ day, but as the most important parameter, trend did not decrease, which means that this is not seismogenic. Then, before the second EQ on September 11, there presented a clear precursor a few days before the EQ (both a significant decrease in trend and an increase in dispersion approaching $+2 \sigma$ level and NF). As for the third EQ, the biggest EQ in 


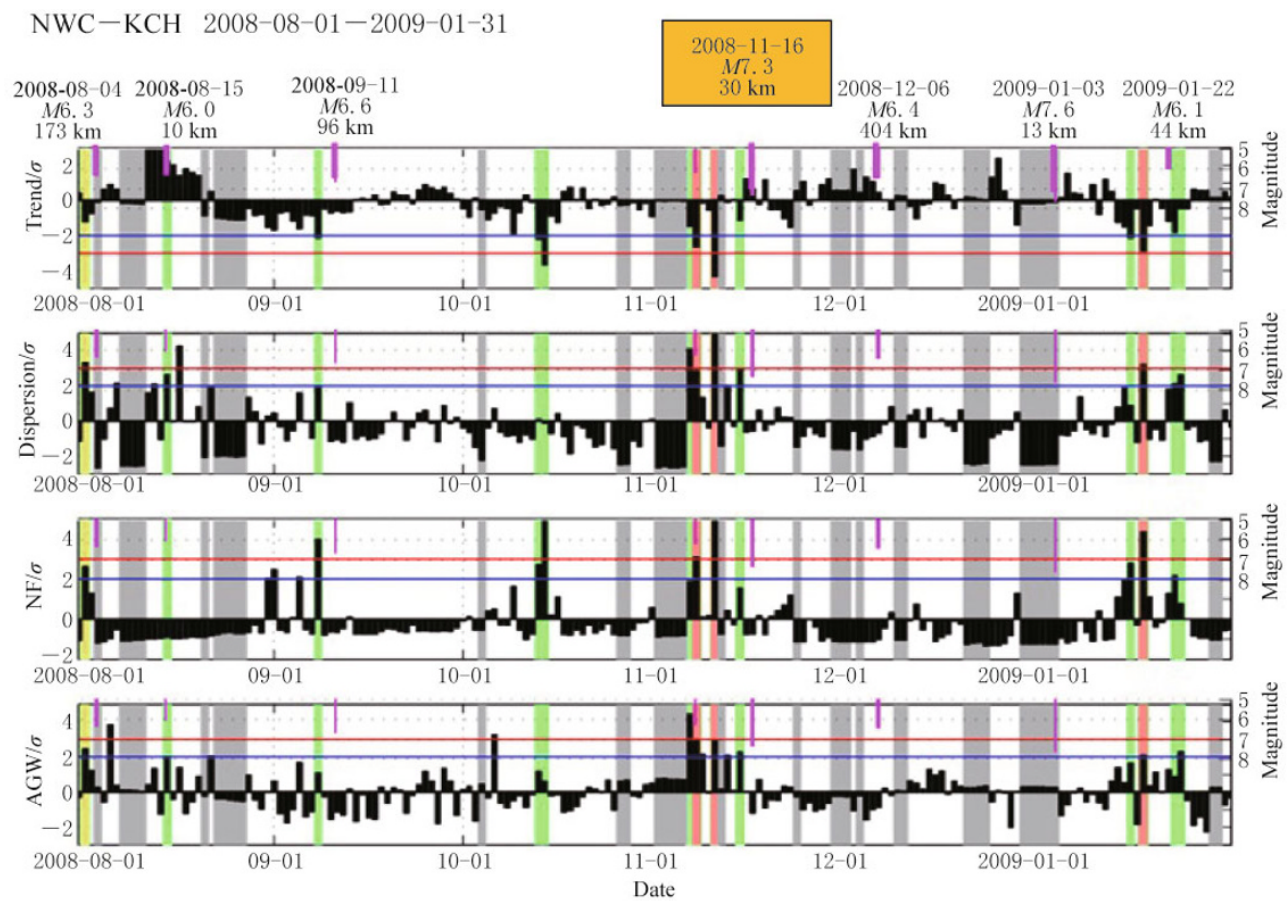

Figure 9 Temporal evolution of the three physical parameters (trend, dispersion, and NF from the top down) for the path from NWC to $\mathrm{KCH}$ and an additional parameter of AGW at the bottom. All quantities are normalized with respect to their corresponding standard deviations. The dates of EQs are indicated on the top and they are indicated by downward purple lines. A red bar means that all of the four physical parameters satisfy their $2 \sigma$ criteria, while a yellow bar means that three physical parameters satisfy their $2 \sigma$ criteria and a green bar means that two physical parameters satisfy their $2 \sigma$ criteria. Grey paths indicate the lack of observation (After Muto et al., 2009b).

this area on November 16, we could find very conspicuous propagation anomalies in two red bars on two days as a series about one week before the EQ, which are highly likely to be precursors of this EQ. You can notice a green box about one month before the EQ on November 16, for which we have observed a significant decrease in the trend, but not so much in dispersion. This additional anomaly is not found to accompany an EQ. We will come to this anomaly later after looking at the same anomaly for other two propagation paths. As for the next EQ on December 6, we could not find out any conspicuous propagation anomaly. This is reasonably acceptable because of a large EQ depth of 404 $\mathrm{km}$, as based on our previous works (Molchanov et al., 1998; Maekawa et al., 2006). Finally, we try to find any anomaly before the last EQ on January 3, which is strong enough $(M=7.6)$ and shallow enough. However there is no propagation anomaly, which is due to the fact that the EQ epicenter is far away $(>700 \mathrm{~km})$ from the propagation path.

Similar detailed discussions are given for other propagations from NWC to MSR and from NWC to
CHF (Kasahare et al., 2010).

Finally, the following characteristics are summarized for several EQs occurred in the Asian region.

1) The nighttime fluctuation method is found to be useful to identify seismo-ionospheric perturbations on the basis of medium-distance (6-8 Mm) subionospheric propagation data even in the NS propagation (for which TT method is not so effective). An anomaly is characterized by a decrease in trend and enhancements in dispersion and NF, just like for short-distance properties in subsection 4.1.

2) When the EQ epicenter is located within the wave sensitive area (or the fifth Fresnel zone) of the propagation path, we can definitely detect a propagation anomaly when the EQ magnitude is greater than 6.0 and its depth is shallow (10 km in this paper). The anomaly appears only as a precursor without any aftereffect.

3) When there occurs a huge EQ with magnitude of 6.6, but its depth is rather large, $96 \mathrm{~km}$, there appears a propagation anomaly on one path, but no anomaly is seen on other propagation paths. This combination of 
EQ parameters (magnitude and depth) seems to be the boundary of exhibiting the VLF anomaly.

4) The main EQ on November 16 showed the following propagation characteristics. This EQ is extremely large with magnitude of 7.3 and also very shallow $(30 \mathrm{~km})$. The resulting anomaly is characterized by a prolonged (about one week) precursory signature about one to two weeks before the EQ. Together with this, it seems that there is an after-effect. Further, the effect of Earth's tides (to be discussed in subsection 4.4) seems to appear one month before this EQ.

These characteristics of possible seismo-ionospheric perturbations would be of essential importance in studying further details of the spatial/temporal properties of seismo-ionospheric perturbations and then in studying their generation mechanism in term of the concept of lithosphere-atmosphere-ionosphere coupling (Molchanov and Hayakawa, 2008; Hayakawa, 2009a).

\subsection{The fluctuation spectra in subionospheric VLF/LF data}

Based on the idea that the fluctuation (or modulation) spectra in the subionospheric VLF/LF data would offer a useful information on the physical process involved in the lithosphere-ionosphere coupling (in other words, the generation mechanism of seismo-ionospheric perturbations), we present our latest result on the AG$\mathrm{W}$ modulations in the VLF/LF data (Muto et al., 2009b), and then we will present our results on the effect of Earth's tides in seismo-electromagnetic effects (Hayakawa et al., 2009) in the next subsection 4.4.

First we discuss the AGW effect. So the purpose of this part is to deal with a big EQ named Miyagi-Oki EQ happened on August 16, 2005 (with magnitude of 7.2 and with depth of $36 \mathrm{~km}$ ) and to investigate further AGW effects in seismo-ionospheric perturbations. We have already studied the ionospheric perturbations extensively for this EQ by means of ground-based VLF observation (Rozhnoi et al., 2007; Muto et al., 2009a) and also by Demeter satellite observation (Rozhnoi et al., 2007). The main emphasis here is to investigate the AGW modulation in VLF/LF data for this Miyagi-Oki $\mathrm{EQ}$ and to obtain further evidence on the importance of this AGW influence on seismo-ionospheric perturbations.

We have used the time period of four months from June 1, 2005 to the end of September, 2005 including the date of the famous and large Miyagi-Oki EQ. This EQ is large enough for us to expect any seismo-ionospheric effect (Muto et al., 2009a). Table 3 summarizes the EQs (with magnitude greater than 5.5, depth smaller than $100 \mathrm{~km}$ ) during the above period which might be relevant to the propagation anomalies observed at different observing stations.

Table 3 EQs used and their focal parameters

\begin{tabular}{|c|c|c|c|c|c|}
\hline Date & Lat. $/{ }^{\circ} \mathrm{N}$ & Long. $/{ }^{\circ} \mathrm{E}$ & Location & Depth/km & Magnitude \\
\hline 2005-06-19 & 35.6 & 140.5 & Chiba & 48 & 5.7 \\
\hline 2005-07-09 & 33.4 & 140.8 & Coast of southern Chiba & 55 & 5.8 \\
\hline $2005-07-23$ & 35.5 & 140.0 & Tokyo bay & 61 & 6.1 \\
\hline $2005-07-27$ & 33.3 & 142.3 & Coast of southern Chiba & 55 & 5.8 \\
\hline 2005-08-01 & 47.0 & 153.9 & Russia & 16 & 5.7 \\
\hline 2005-08-07 & 36.3 & 141.4 & Coast of Ibaraki & 39 & 5.5 \\
\hline 2005-08-10 & 48.7 & 158.1 & Russia & 31 & 5.5 \\
\hline 2005-08-16 & 38.3 & 142.0 & Coast of Miyagi & 36 & 7.2 \\
\hline 2005-08-24 & 38.6 & 143.0 & Coast of Miyagi & 10 & 6.1 \\
\hline 2005-08-30 & 38.5 & 143.2 & Coast of Miyagi & 21 & 6.1 \\
\hline
\end{tabular}

The LF JJY transmitter in Fukushima prefecture, is used again in Figure 10. The data from three observing stations (Kamchatka (KCK), Moshiri (MSR) and Kochi (KCH) indicated by stars) are analyzed. The EQs in Table 3 are plotted in Figure 10. As the wave sensitive area for each propagation path, we have used the fifth Fresnel zone for each path.

Figure 11 illustrates the raw data on the observation of amplitude (intensity) of subionospheric LF prop- agation from the JJY transmitter, which is received at above-mentioned three stations in Figure 10. The left refers to JJY-KCH path, the middle, JJY-MSR and the right, JJY-KCK. The date of Miyagi-Oki EQ of our concern is August 16 as indicated by EQ in Figure 11. The date goes upward from the bottom (August 1st) to the top (August 22nd). As summarized already in subsection 4.1, the trend decreases before the EQ, and also the NF is greatly enhanced before the EQ. This 


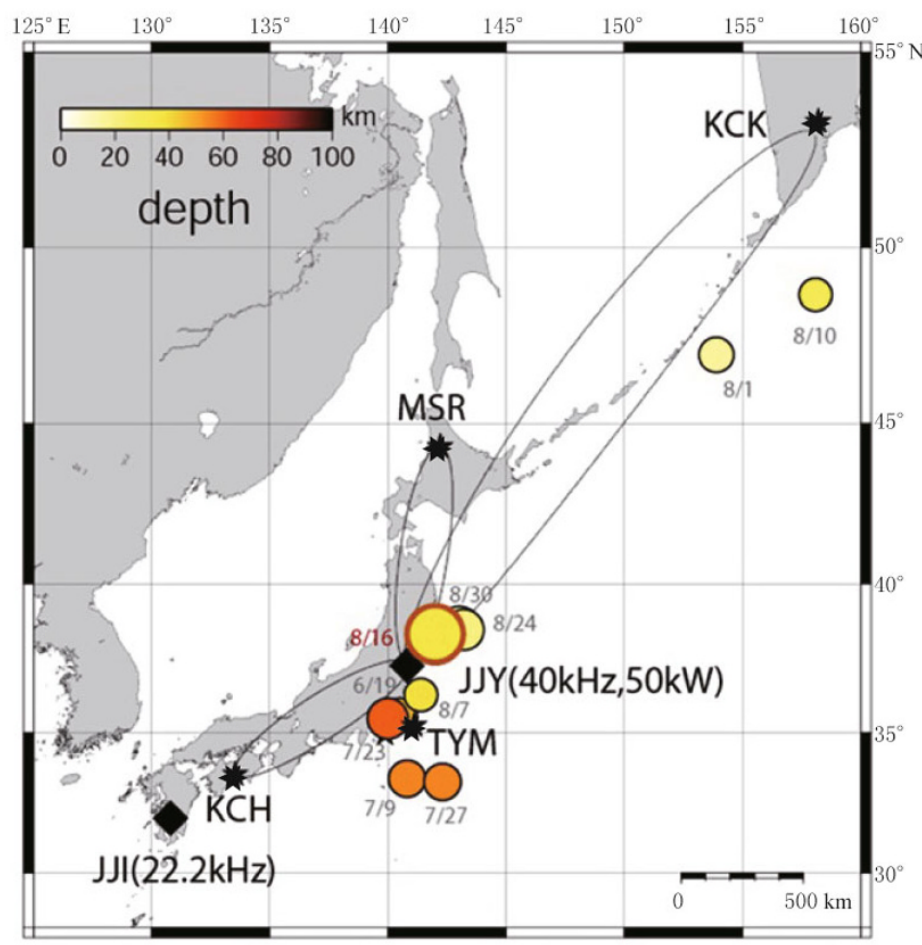

Figure 10 Locations of our JJY transmitter indicated by a diamond and receiving stations (Moshiri (MSR), Kamchatka (KCK) and Kochi $(\mathrm{KCH})$ ) indicated by stars (some other information is also included, JJI transmitter (diamond) and TYM (Tateyama, Chiba) station, but not used in this study). EQs treated are plotted as circles, for which the center is the epicenter, its radius is proportional to magnitude, and its depth is indicated by color.
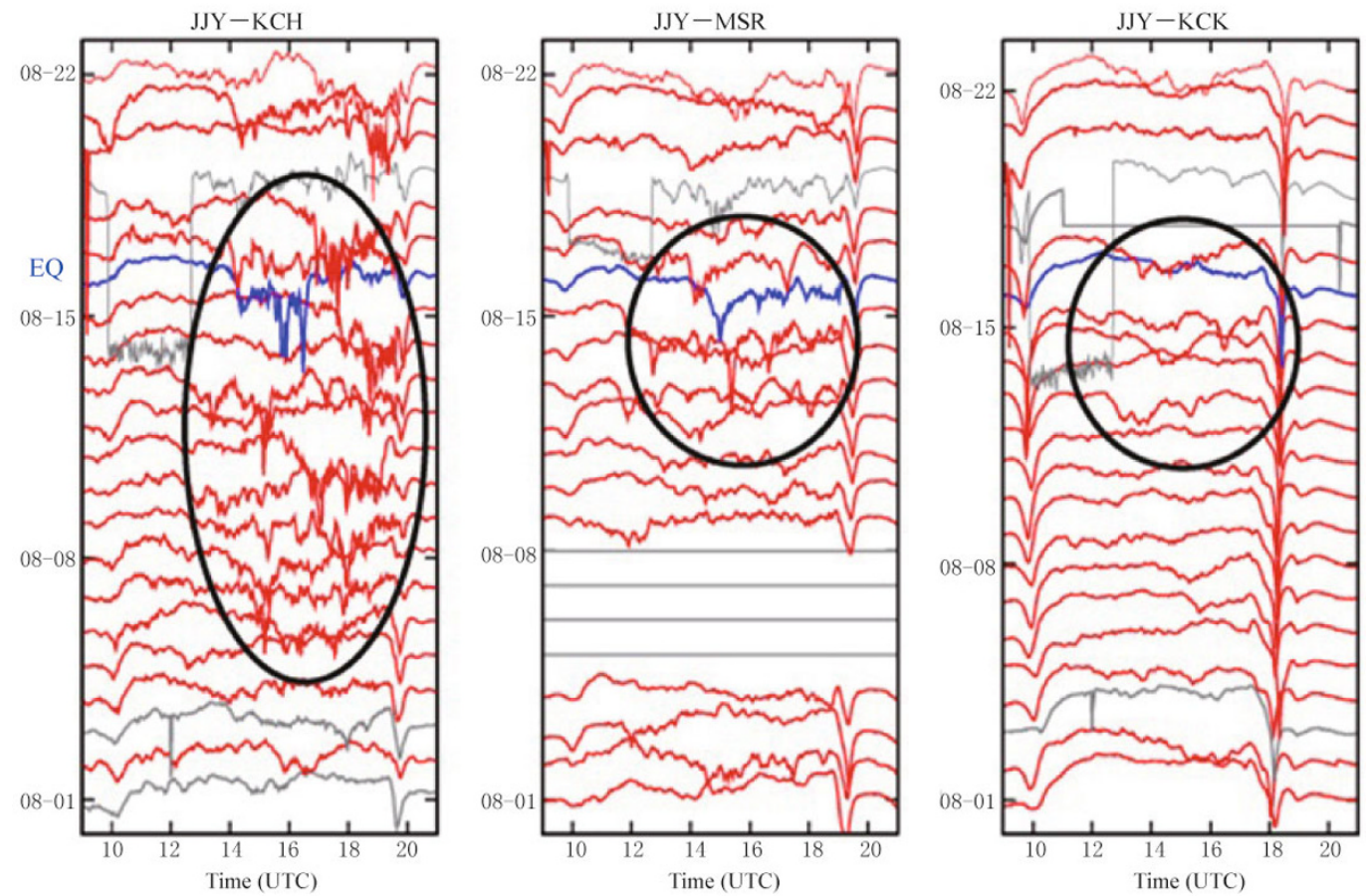

Figure 11 Temporal evolutions of the diurnal variation of signal intensity observed at three stations in August of 2005. Date goes upwards from August 1 (bottom) toward August 22 (top), with the EQ day indicated by EQ (August 16). Time is given in UTC (so that LT in Japan is UTC+9h). 
is qualitatively consistent with our previous finding (Maekawa et al., 2006).

Here we define the two physical quantities for our analysis. We use the data during local night between the TT evening and TT morning in Figure 11. First, we estimate the value of $\mathrm{d} A(t)=A(t)-<A(t)>$ as shown in Figure 12a as explained before. By using this residue $\mathrm{d} A(t)$ (shown by black curve in the bottom of Figure 12a), we have already defined (1) trend, (2) dispersion and (3) NF in subsection 4.1. In addition to these physical quantities, we propose an additional use of the AGW influence as a new physical quantity. Figure 12 shows an example of how to estimate the fluctuation spec- tra. First, we perform the FFT analysis on $\mathrm{d} A(t)$ and we obtain the fluctuation spectrum in a wide frequency range of AGW (period $T=1-10 \mathrm{~min}$ ) and acoustic wave (AW) $(T=10-100 \mathrm{~min})$ in Figure 12b. We then define $\mathrm{d} S(f)=S(f)-<S(f)>$, in which $S(f)$ is the fluctuation spectrum for one particular day (red curve) and $<S(f)>$ is the average spectrum averaged over -30 to -1 day of the current day (30 to 1 day before the current day) (blue curve). $\mathrm{d} S(f)$ is indicated by a black bar in Figure $12 \mathrm{~b}$, and we take only positive $\mathrm{d} S(f)(\mathrm{d} S(f)>0)$ for our interest and the AGW modulation $(M)$ is defined as follows.
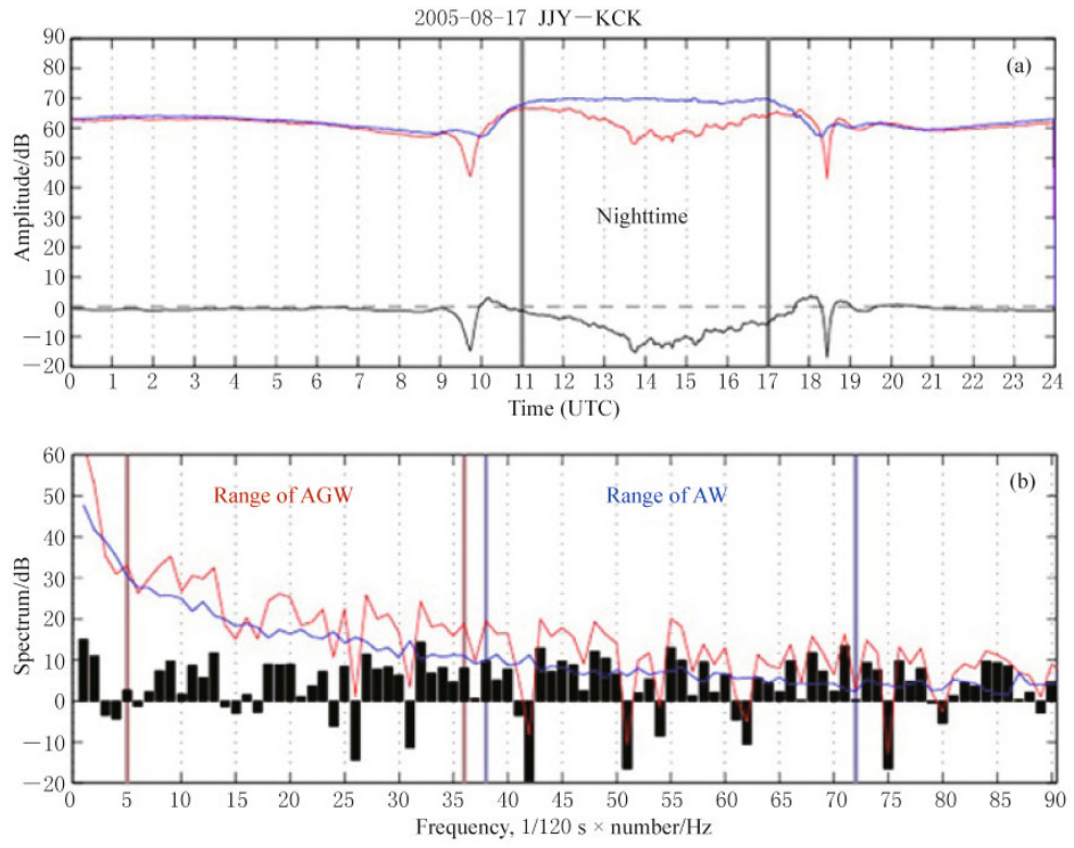

Figure 12 An example of estimating $\mathrm{d} S(f)$ and AGW modulation $(M)$ index by means of FFT analysis for $\mathrm{d} A(t)$. (a) The diurnal variation of $\mathrm{d} A(t)(=A(t)-<A(t)>)$ (in black) and nighttime part (UT=12-16 h) used for the estimation of $\mathrm{d} S(f)$. (b) The fluctuation spectrum of nighttime $\mathrm{d} A(t)$ (red curve stands for fluctuation spectrum on the particular day and blue curve, the average spectrum over -30--1 day of the current day) and the difference (or residue) $\mathrm{d} S(f)$ plotted by black bars. The range of AGW is indicated with two limiting values ( $T=100 \mathrm{~min}$ and $10 \mathrm{~min}$ as vertical red lines) together with the range of acoustic waves (AW, $T=1-10$ min), and we take the positive $\mathrm{d} S(f)$ to define the AGW $M$ index.

$$
\text { AGW } M=\frac{\int_{\mathrm{AGW}} \mathrm{d} S(f) \mathrm{d} f}{\text { Range (in frequency) of AGW }}
$$

where $\mathrm{d} S(f)$ is plotted against frequency in Figure 12, and AGW range is limited by two vertical red lines (left, AGW period $T=100 \mathrm{~min}$ and right, $T=10 \mathrm{~min}$ ).

Finally we impose the normalization for these physical quantities (trend, NF and AGW $M$ ), in order to delete any long-term (e.g. seasonal) effects in the following way.

$$
\text { DATA }^{*}=\frac{\text { DATA }-\overline{\mathrm{DATA}}}{\sigma}
$$

where DATA will be one of the quantities (trend, NF and AGW $M$ ), and $\overline{D A T A}$ indicates the corresponding mean value of each quantity averaged over $-1--30$ days 
of the current day, and $\sigma$ is the corresponding standard deviation.

We are ready to show the analysis results, but we illustrate only one example of the propagation path, JJY-KCK, because other paths (JJY-MSR and JJY$\mathrm{KCH}$ ) have been described in Muto et al. (2009b). We take Figure 13 as an example of presentation. From the top, we find the temporal evolution of NF, the middle, trend, and the bottom, a new parameter of AGW $M$.
All values are normalized by their corresponding standard deviations as given in the above equation (that is, DATA*). At the top of each panel we find downward red bars, which indicate the times of EQs with length corresponding to the EQ magnitude. Only EQs with magnitude greater than 5.5 are plotted, which are found to lie within the fifth Fresnel zone (wave sensitive area) of the propagation path.

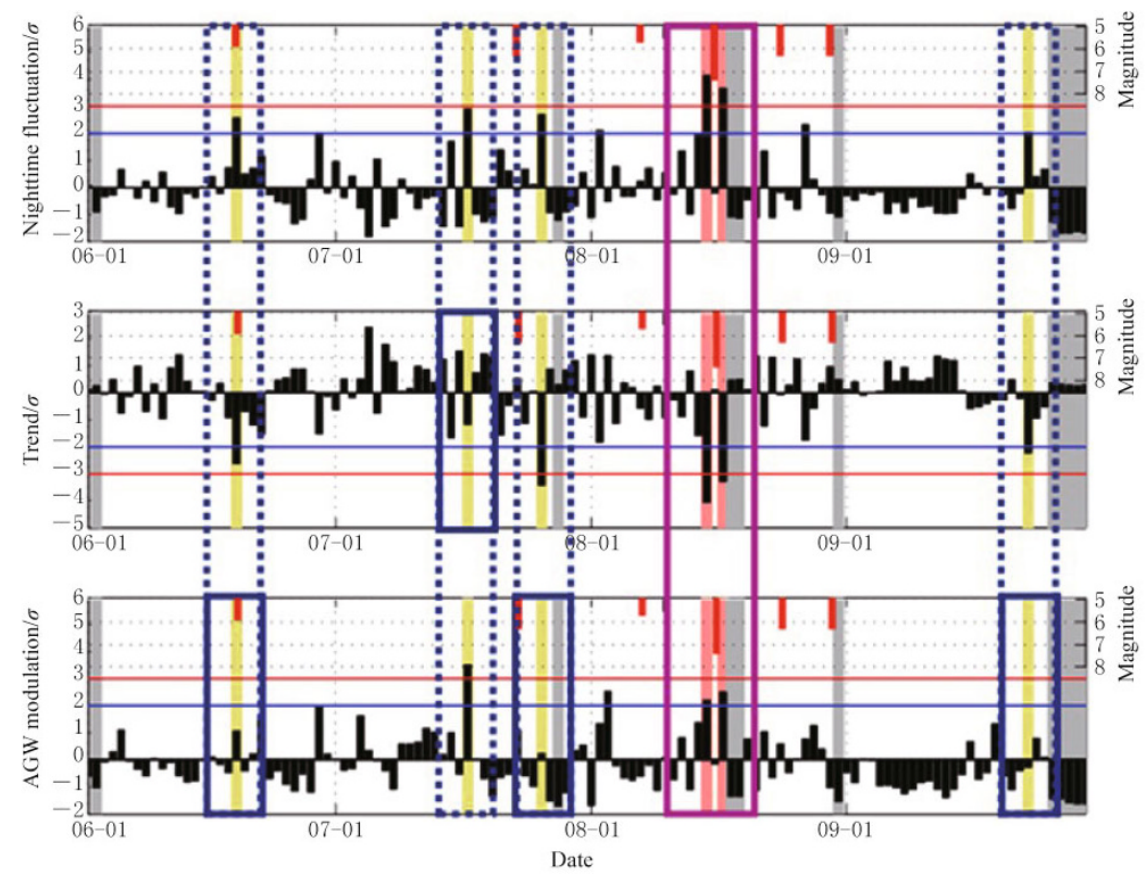

Figure 13 Temporal evolutions of three physical parameters (NF, trend, and AGW $M$ index) during four months including the date of Miyagi-Oki EQ on August 16, 2005. All of the three quantities are normalized by their corresponding standard deviations $(\sigma)$ (during -30 to -1 day of the current day). Downward red bars on the top of each panel indicate the times of EQs with length indicating the magnitude. You notice one red box and four blue dotted boxes. In the red box you can find two red vertical bars and red means that all of three physical quantities satisfy their criteria, suggesting that these peaks are seismogenic as a precursor and an after-effect of the EQ. Boxes in dotted blue line mean that there exist an anomaly indicated by vertical yellow bars, but two of the three parameters satisfy the $2 \sigma$ criteria (unsatisfaction of one parameter is shown by a full blue box). Gray zone means the period of no observation, so that some values for these grey zones are meaningless. After Muto et al. (2009a).

First of all we look at the time period just around our target EQ, Miyagi-Oki EQ. Just around the MiyagiOki EQ, we have found two vertical red bars surrounded by a box in red. A few days before this EQ, we have found that the trend decreases below $-2 \sigma$ line and also the NF exhibits an increase above $2 \sigma$ line. Together with these, it is found that the third quantity of AGW $M$ index introduced here would offer additional confirmation on the importance of AGW modulation. Another red bar just after this EQ is also characterized by both a significant decrease in trend (exceeding $-2 \sigma$ ), an increase in NF (exceeding $2 \sigma$ ), and the AGW $M$ index well exceeding its $2 \sigma$ line. Red means that three parameters satisfy the criteria.

We have found that this AGW $M$ index is significantly increased exceeding the $2 \sigma$ criteria for all of the three propagation paths, JJY-KCK, JJY-MSR and JJY-KCH. This fact means that the AGW fluctuations are extremely enhanced for a large EQ, which would lend us a further support to the mechanism, second 
channel (AW and AGW channel) in the lithosphereionosphere coupling mechanism to be discussed later. Furthermore, the simultaneous increase in AGW $M$ index can be used as a definite confirmation of the presence of seismo-ionospheric perturbations.

\subsection{The effect of Earth's tides on seismogenic phenomena}

Next we discuss, for the first time, the effect of Earth's tides in seismogenic electromagnetic phenomena. In order to convince the people of the presence of electromagnetic effects highly likely to be associated with an EQ, we have made an attempt to find out the effect of Earth's tides in seismogenic phenomena (Hayakawa et al., 2009). If this effect is evident, we can conclude for sure that the relevant phenomenon is really related to an $\mathrm{EQ}$.

It has been debated for years whether Earth tides trigger EQs. A recent paper by Tanaka et al. (2004) has been published on a statistical study of tidal triggering, which has proven the significance of these Earth tides in EQ's triggering. Following this study, Tanaka (2006) has provided some more evidences that the EQs occurred near the epicenter of the 2004 Sumatra EQ were affected by Earth tides for the period of 10 years before the main shock. Sue (2009) has then shown the evidences of apparent tidal effects in EQ triggering in the three specific regions in Japan such as the Sagami trough region by paying particular attention to some specific fault structures.

EQs are definitely a mechanical effect, so that it is not so difficult to imagine that the Earth tide is a possible candidate of triggering large EQs when the focal zone is self-organized to a critical or a super-critical stage. On the other hand, there have recently been accumulated a lot of evidences on the presence of precursory electromagnetic effects of EQs, and we can list a few examples. The ULF electromagnetic emissions as the first example are believed to be generated near the EQ focal zone and to have propagated up to the Earth's surface (e.g., Fraser-Smith et al., 1990; Kopytenko et al., 1993; Hayakawa et al., 1996a). Molchanov and Hayakawa (1995) proposed a mechanism of microfracturing for seismogenic ULF emissions. Whatever the mechanism is microfracturing or electrokinetic (see Molchanov and Hayakawa (2008) for details), and these ULF emissions are fundamentally based on any mechanical effect in the EQ focal zone. The next phenomenon clearly associated with EQs is the ionospheric perturbations as discussed extensively in this review. The mechanism how and why the ionosphere is per- turbed prior to an EQ is not well understood, though we have proposed a few hypotheses (Hayakawa, 2004; Molchanov and Hayakawa, 2008) and will discuss them later in subsection 4.6. No matter which mechanism is most plausible, the initial agent of seismogenic phenomena is located near the EQ focal region and it is just a mechanical effect (not macroscopic, but probably microscopic).

Therefore, we try to find out any modulation of Earth tides in various kinds of seismogenic phenomena. This study would be a bridge between the seismology and our seismo-electromagnetics, and it would offer an important additional confirmation on the presence of seismogenic electromagnetic effects.

The Earth tides of the Moon and the Sun affect the occurrence of EQs. This is the phenomenon which appears only when Earth tides deform a fault from the direction to assist the fault slipping (and the corresponding seismogenic electromagnetic effects). Thus we investigate if there are any effects of the specific lunar phase in electromagnetic EQ precursors. The lunar phase is explained in Figure 14. For an index of the lunar phase, it is possible to apply the ecliptic longitude difference between the Moon and the Sun of the ecliptic coordinate system. This is the angle with a numerical value of 0 to 360 degrees: new moon at $0^{\circ}$, first quarter moon at $90^{\circ}$, full moon at $180^{\circ}$, and last quarter moon at $270^{\circ}$. Since the Moon orbits the Earth in 29.5 days on average, the average traveling speed of the Moon is 12.2 degrees per day. We here use the number of days with respect to such specific lunar positions as the new, first

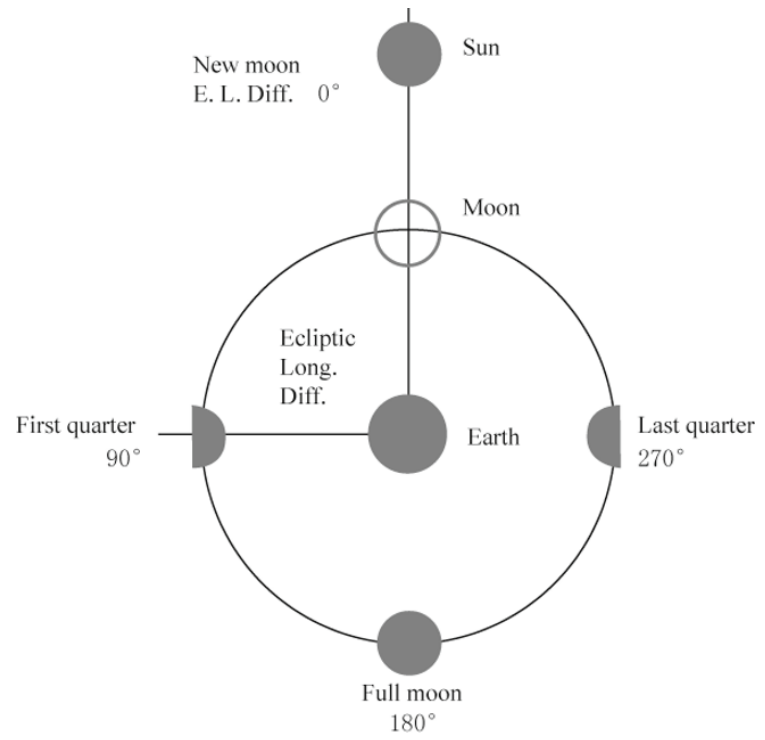

Figure 14 Explanation of lunar phase $\left(0^{\circ}\right.$, new moon, $90^{\circ}$, first quarter, $180^{\circ}$, full moon and $270^{\circ}$, last quarter). 
quarter, full and last quarter moon as the indicator of lunar phase.

We investigate the effect of Earth tides in different phenomena of ULF geomagnetic anomalies, VLF propagation anomalies, and radio emission anomalies at different frequencies. We study these phenomena one by one in the following.

The reports of ULF geomagnetic anomalies before large EQs have been summarized in Hayakawa et al. (2007a). The ULF signal observed at any particular station is known to be composed of different noise sources including the dominant geomagnetic variation (geomagnetic pulsations), artificial noise and seismogenic noise as our target. So it is very difficult to find any relationship to the lunar phase when we use the raw data, and it is of essential importance to perform any pre-processing to the raw data so as to distinguish our seismogenic noise from the whole signal or to enhance our seismogenic signal against the other noises. Therefore a few possible methods have been developed for this purpose. The first is called "polarization" analysis, in which the use of the ratio of the vertical $(Z)$ to horizontal $(H)$ magnetic field components allows us to identify the seismogenic effect against the space noise (geomagnetic variation) (Hayakawa et al., 1996a). The second is the use of principal component analysis (PCA), in which a multiple of stations of simultaneous observation enables us to distinguish a few possible noise sources such as (1) geomagnetic variation, (2) man-made noise, and (3) seismogenic emission (Gotoh et al., 2002). One more important point to mention as regards the seismogenic ULF emissions is their typical temporal evolution. They show the first peak one to two weeks before the EQ, becomes quiet in amplitude several days before the EQ and exhibits an abrupt increase a few days before the EQ (Fraser-Smith et al., 1990; Kopytenki et al., 1993; Hayakawa et al., 1996a).

We here deal with our former two ULF events: the 1993 Guam EQ and the 2000 Izu islands EQ swarm in the following.

\subsubsection{Case 1: The 1993 Guam EQ (M8.0)}

The Guam EQ (M8.0, depth $59 \mathrm{~km}$ ) occurred on August 8, 1993 (Hayakawa et al., 1996a). ULF geomagnetic anomalies were observed for this EQ, but the relationship with Earth tides could not be identified in the raw data due to the dominant contribution by the geomagnetic variation. With the polarization analysis shown in Figure 15, the ratios of $Z / H$ have exhibited the following characteristics (Hayakawa et al., 1996a). The main point of Hayakawa et al. (1996a) is that we observed an enhanced $Z / H$ during one month before the EQ, which is likely to reflect the occurrence of seismogenic emissions. The enhanced $Z / H$ lasts for about one month, so that we take the EQ day as a reference point, three days before the last quarter moon as shown as LQ-3d in the figure. The days of the same lunar phase are marked ( $-1 \mathrm{mo},-2,-3$ and -4 (mo: month)) for the period of four months before the EQ in the figure. We note that the period between the same lunar phases is 29.5 days on average (a lunar month), which is the duration of the moon's orbiting the Earth. The value of $Z / H$ is increased two months before the EQ, further it takes a maximum (at $-1 \mathrm{mo}$ ) at the same lunar phase as that of the EQ and minima (shown in broken lines) in the middle. There are several small oscillations with the different lunar phases, suggesting overlapping of other patterns with different lunar phases. As the summary, the behavior of $Z / H$ shows a clear maximum-minimummaximum pattern synchronizing with the lunar phase of the EQ during two months before its occurrence.

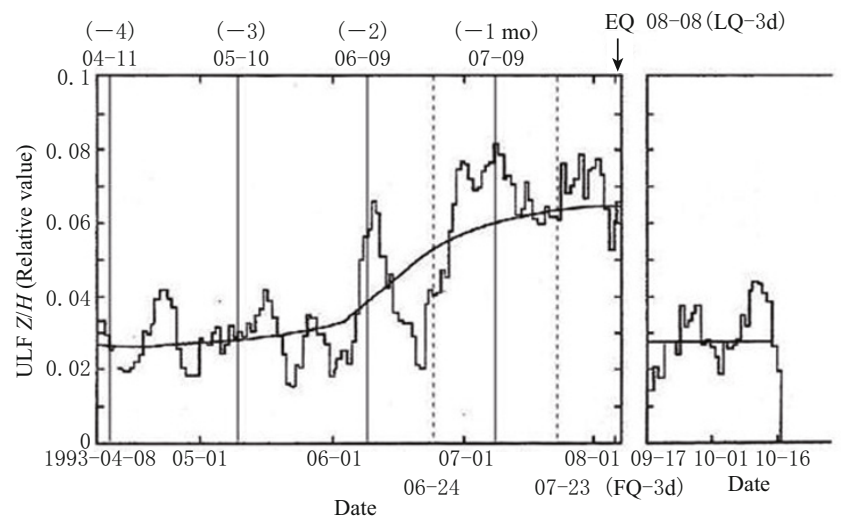

Figure 15 Lunar phase analysis for the polarization $(Z / H)$ of ULF geomagnetic emissions (at the frequency of $0.01 \mathrm{~Hz}$ ) for the 1993 Guam EQ (M8.0). After Hayakawa et al. (2009).

\subsubsection{Case 2: The $2000 \mathrm{Izu}$ islands EQ swarm (M6.4 etc.)}

From June to August in 2000 there were observed a series of large tectonic activities which included a total of more than 3000 EQs with five large EQs (M6.4 on July $1, M 6.1$ on July 9 , M6.3 on July $15, M 6.4$ on July 30, and $M 6.0$ on August 18) and five eruptions at Miyake island. This activity was caused by intrusion of magma and such EQ activities are the response to the stress rate. The ULF electromagnetic radiation has already indicated anomalies for this activity (Gotoh et al., 2002; Uyeda et al., 2002). The PCA method (Gotoh 
et al., 2002) enabled us to separate a few noise sources. The first principal component (whose eigenvalue is $\lambda_{1}$ ) is found to correspond very well to the geomagnetic variation, the second principal component $\left(\lambda_{2}\right)$ is then found to reflect the human activity, so that the second component is due to the man-made noise. The last and the weakest principal component $\left(\lambda_{3}\right)$ might include the seismogenic effect if it exists. The upper panel of Figure 16 shows the temporal evolution of the third principal (weakest) component $\lambda_{3}$ at the frequency of $0.01 \mathrm{~Hz}$ from February 1 to July 16, 2000, which is taken from Gotoh et al. (2002).

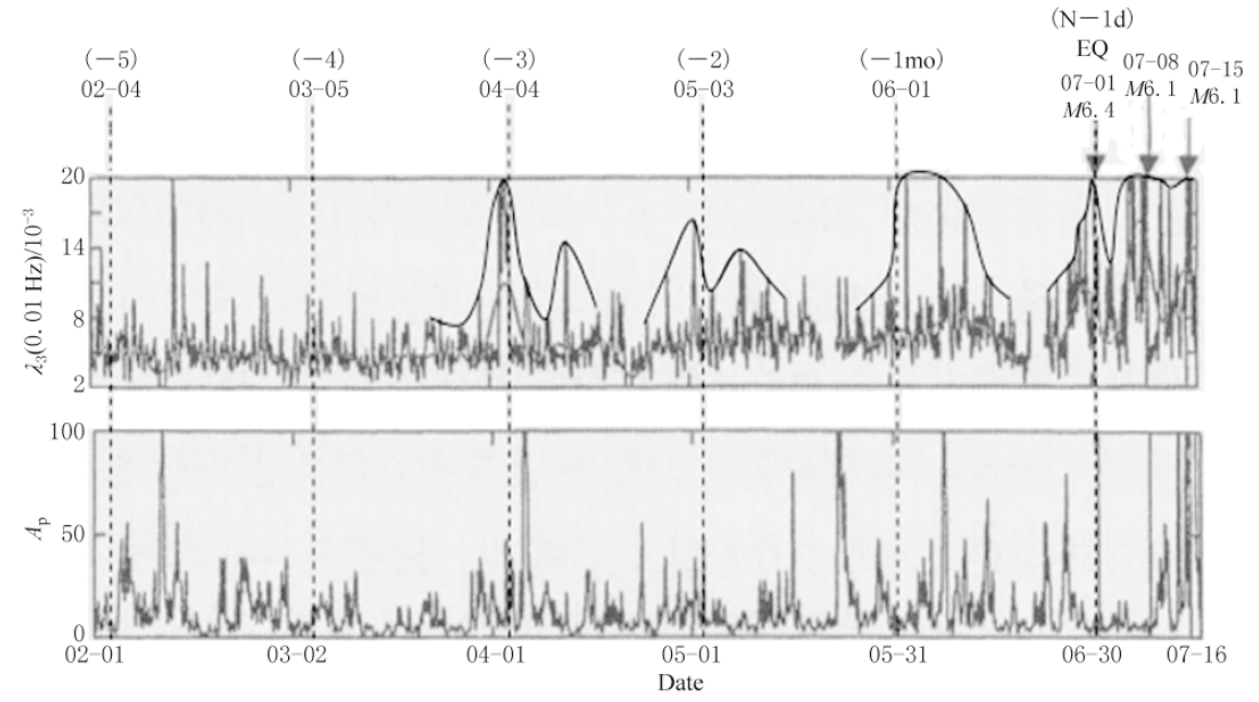

Figure 16 Lunar phase analysis of the third principal component (its eigenvalue, $\lambda_{3}$ ) of geomagnetic variation at the frequency of $0.01 \mathrm{~Hz}$ (upper panel) and geomagnetic activity, $A_{\mathrm{p}}$ index (lower panel) for the 2000 Izu islands EQ swarm (M6.4 etc.). After Hayakawa et al. (2009).

The largest EQ (M6.4) of the activity occurred on July 1 is shown in Figure 16, and this day is taken as a reference point corresponding to a new moon -1 day which is shown as $\mathrm{N}-1 \mathrm{~d}$ in the figure. The days of the same lunar phase as well as the envelope curves connecting peaks are indicated for the period of 5 and 3 months respectively. From the beginning of April which is three months before the EQ, there are apparent increases of the eigenvalue $\lambda_{3}$ of the third principal component at the same lunar phase as that of the EQ and the decreases in the middle. Note that the geomagnetic activity $A_{\mathrm{p}}$ shown in the lower part of Figure 16, does not show such a tendency. In summary, the behavior of $\lambda_{3}$ shows a maximum-minimum-maximum pattern synchronizing with the lunar phase of the EQ during three months before its occurrence.

Next we move on to the VLF/LF propagation anomaly. Though having studied several events, we only present the result for the Kobe EQ. Figure 17 shows the temporal evolutions of TT phase (upper panel) and amplitude (lower panel) for a period of 1.5 months before and after the Kobe EQ, which is taken from Hayakawa et al. (1996b) and already discussed in subsection 3.1.
Figure $17 \mathrm{a}$ shows " $\Delta t_{\mathrm{e}}$ " (in unit of ph), which is the variation of the evening TT (phase), where the standard deviation $\sigma$ is that for one month period. The figure shows that on January 14, which is three days before the EQ, there was a large deviation exceeding $2 \sigma$. The deviation exceeding $2 \sigma$ is an unusual phenomenon, while there were no reports of so extensive geomagnetic activity, solar activity and rainfall in the Kobe region as to affect the VLF transmission (see Hayakawa et al., 1996b; Molchanov et al., 1998). Thus the phenomenon was found to be likely to be a precursory signal of the EQ occurred immediately afterward (Hayakawa et al., 1996b; Molchanov et al., 1998), as discussed in section 3.

The day of shift in TT exceeding $2 \sigma$ is chosen as a reference point in Figure 17. The day was January 14 (three days before the EQ) which was three days before a full moon day and is shown as F-3d in the figure. The same lunar day of one month before was December 15 (shown by a vertical thick line) and the day of the middle between the two (shown by a vertical dotted line) which is a new moon -3 days, was December 29. The value $\left(\Delta t_{\mathrm{e}}\right)$ firstly took a maximum three days before 


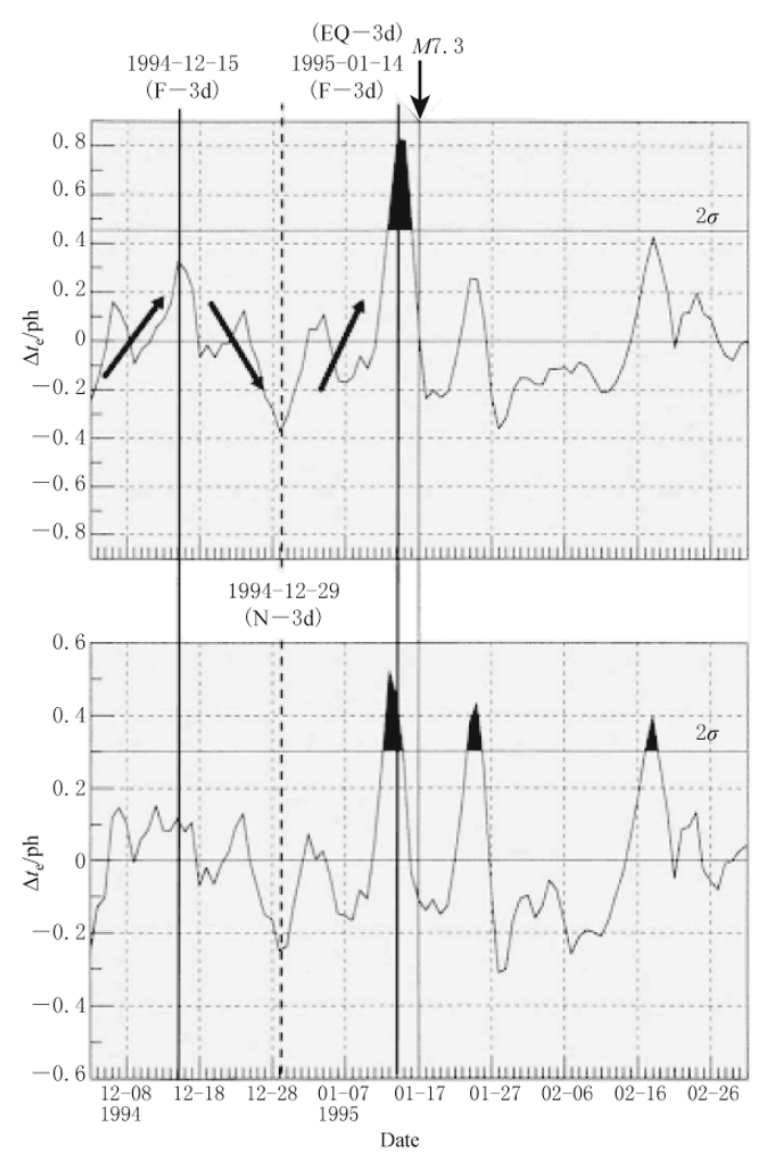

Figure 17 Lunar phase analysis of phase (upper) and amplitude (lower) for the evening TT for the 1995 Kobe earthquake (M7.3). After Hayakawa et al. (2009).

a full moon $(\mathrm{F}-3 \mathrm{~d})$, then decreased to a minimum three days before a new moon $(\mathrm{N}-3 \mathrm{~d})$, and raised again to take a maximum three days before the EQ. And it finally decreased to zero, then the EQ occurred. During this period, we observed some oscillations with the period of 9-10 days on the basis of spectrum analysis. The deviation from the average increased with time, so the last maximum was the largest and was regarded as a conspicuous precursory to the EQ. The lunar phases at the maximum and minimum of $\Delta t_{\mathrm{e}}$ are found to coincide or to be exactly opposite with that of the reference point. We conclude that there was detected a clear maximum-minimum-maximum pattern during one month before the EQ, and the deviation seems to increase with time toward the EQ (thus the last maximum was the largest). After passing the last maximum, the $\Delta t_{\mathrm{e}}$ values decreased (or relaxed) and several days later the EQ occurred when the deviation became nearly zero.

Finally we discuss the effect of Earth's tides for the same Kobe EQ, but different kinds of electromagnetic

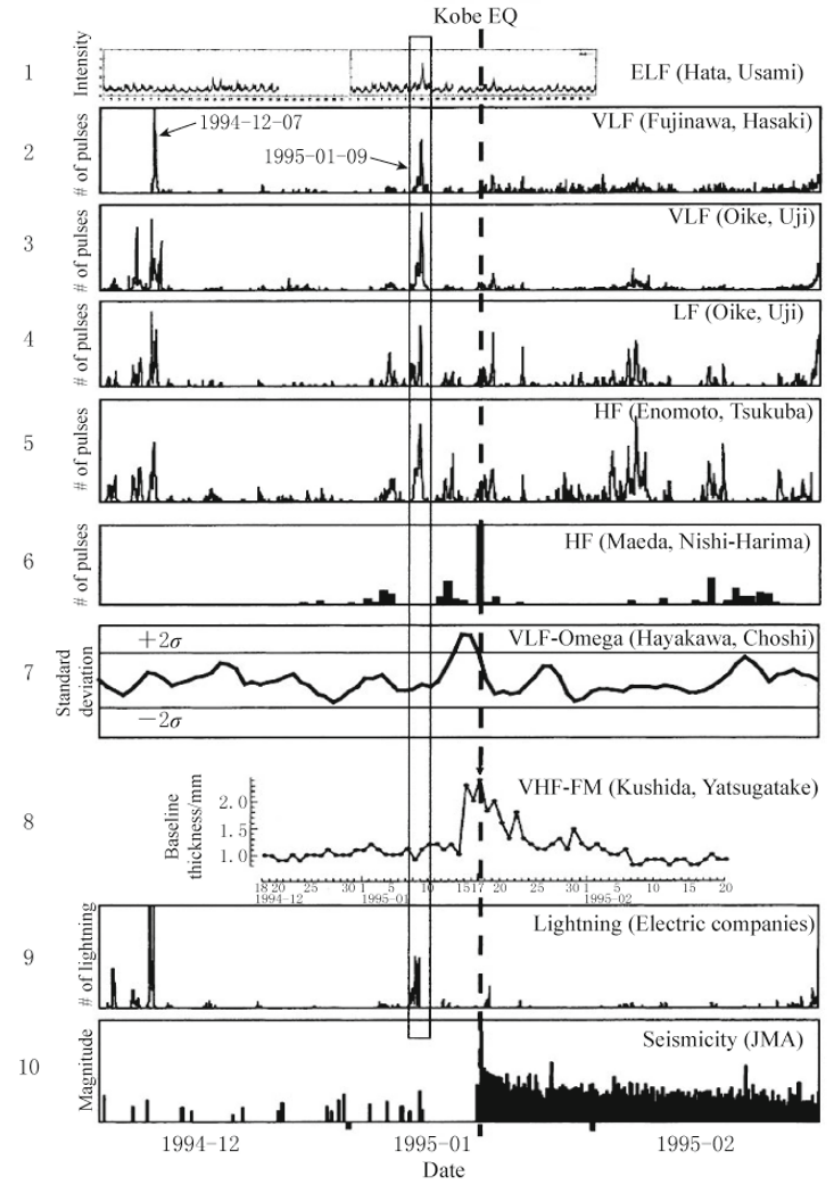

Figure 18 Electromagnetic phenomena for the Kobe EQ on January 15, 1995. Radio emissions at ELF (Row $1)$, VLF (2 and 3), LF (4), HF (5 and 6), subionospheric VLF (Omega (Tsushima)-Inubo) (7), over-horizon VHF (8), lightning (9) and seismicity (10). The January 9 is an anomalous day (marked by a box). After Hayakawa et al. (2009) and Nagao et al. (2002).

observations for this EQ. Many types of anomalies were reported for the Kobe EQ, and Figure 18 is a summary of those different phenomena (Nagao et al., 2002). The figure shows that on January 9, 1995 that is eight days before the EQ, anomalies of ELF, VLF, LF and $\mathrm{HF}$ radio emissions as well as atmospherics (indicated by lightning) were observed. These phenomena are indicated by a box, and they are thought as precursors to the EQ. On December 7, 1994 that is one month before January 9, there were also observed anomalies of VLF, LF and HF radio emissions as well as atmospherics. The first anomaly appeared on January 9, which corresponds to a first quarter moon. The same lunar phase day of one month before is December 10 . So the anomalies in December and January are found to have occurred un- 
der the similar lunar phases. Besides the measurements show a maximum-flat-maximum pattern, which is similar to the measurements of VLF in the previous subsection. We have studied a few categories of seismogenic effects, and the following findings have emerged.

1) ULF geomagnetic anomalies are found to show a maximum-minimum-maximum pattern in synchronism with the lunar phase of the EQ during several months before its occurrence.

2) VLF/LF propagation anomalies are also found to exhibit the similar variation to that of ULF emissions, but not so clear as compared with the ULF emissions. Sometimes we have found some shift with the lunar phase and more irregularities in the variation. The deviation from the average value is found to increase with time, thus the peak before the EQ is the largest deviation usually exceeding $2 \sigma$. After this peak we observe some oscillations and there will be the occurrence of an EQ around the time when the deviation is just around zero.

3) Radio emissions at different frequencies (ULF, VLF, LF, VHF, etc.) are found to contain the similar tidal modulation.

The lunar tidal effect on EQ triggering is recognized recently in the field of seismology (Tanaka, 2006), in which this effect becomes very apparent only before a large EQ. Similarly, there have been accumulated a lot of evidences on the electromagnetic effects as EQ signatures, which are believed to be very complementary to the EQ mechanical phenomena. The initial agent of our seismogenic electromagnetic effects is definitely any mechanical effect in the lithosphere (or around the EQ focal zone), so that such a tidal effect might be involved even in the seismogenic electromagnetic effects when the lithosphere is just at a critical or at a super-critical stage.

According to Tanaka et al. (2004), the tidal effect is likely to be clearly seen for huge EQs (with larger magnitude) in which the focal zone is well self-organized to a critical or a super-critical stage. We have already known that both of ULF electromagnetic emissions and ionospheric perturbations as seen by VLF signals are only observed for huge EQs (at least with magnitude greater than 6.0 or so) (Hayakawa, 2009a, 2010; Hayakawa et al., 2007a, 2010b), so that we have analyzed our former data satisfying this EQ magnitude criterion in this analysis.

The lithospheric phenomenon of ULF emissions is found to exhibit a rather clear modulation of Earth tides, which means that the ULF emissions observed for the 1993 Guam EQ and 2000 Izu EQ swarm are clearly modulated by Earth tides one and two months before the EQ. This, in turn, leads to the idea that the presence of tidal modulation would be a very strong support to that those emissions are highly likely to be seismogenic and to be attributed to some effect like microfracturing (Molchanow and Hayakawa, 1995) as the mechanical effect (though probably microscopic) in the lithosphere. We have already known that the lithosphere for a huge EQ is well self-organized up to a critical or a super-critical stage by means of former fractal analyses (Hayakawa et al., 1999; Smirnova et al., 2001, 2004; Gotoh et al., 2003, 2004; Ida et al., 2005; Ida and Hayakawa, 2006; Hayakawa and Ida, 2008). Because the focal zone is well self-organized, we are ready to anticipate the generation of different kinds of seismogenic electromagnetic phenomena. Especially, the seismogenic ULF emission is the direct consequence of the lithosphere (mechanical effect (microfracturing)), and this is the reason why we have observed a clear tidal effect. The identification of such a clear tidal effect might offer an important physical indication that those ULF emissions reported before are highly likely to be precursors of EQs.

On the other hand, the VLF/LF data suggesting the presence of seismo-ionospheric perturbations are found to show the similar tidal modulation. However, the effect is not so clear as compared with the case of ULF emissions in such a way that we sometimes find a certain shift with respect to the lunar phase, the presence of some higher frequency modulations. This is easy to understand because the modulation is not direct as in the case of ULF emissions, but it might propagate through the atmosphere up to the ionosphere.

We believe that the above study would offer a bridge between the seismological (or mechanical) effect and our seismo-electromagnetics effect. Also, the presence of tidal effect would be of essential importance in obtaining further confident confirmation of those effects seeming to be an EQ precursor. Of course, it needs to be mentioned that we have to increase the number of events large enough to persuade everybody.

Finally, we would like to add one more recent result by Imamura et al. (2010). Fractal analysis has been applied to the local nighttime data of subionospheric LF propagation, and it is found that when we pay attention to the period just around the EQ, we detect some significant increases in the fractal dimension either in the AW or AGW frequency range. This might indicate that the self-organization effect prior to an EQ in the 
lithosphere might be seen even in the lower ionosphere, probably in terms of atmospheric oscillation effect.

\subsection{Ionospheric perturbations in possible asso- ciation with the 2010 Haiti EQ}

The 2010 Haiti EQ took place at the geographic coordinates $\left(18.45^{\circ} \mathrm{N}, 72.45^{\circ} \mathrm{W}\right)$ at $21: 53: 09 \mathrm{UTC}$ on 12 January, 2010 (16:53 LT). The magnitude of this EQ was $M_{\mathrm{W}} 7.0$ and the depth was $10 \mathrm{~km}$; these conditions are extremely favorable for inducing ionospheric perturbations (e.g., Hayakawa et al., 2010b). The epicenter was located about $15 \mathrm{~km}$ west-south-west of the Haiti's capital of Port-au-Prince. This area is characterized by the proximity to the collisional boundary between the North American and Caribbean plates, where we expect very often shallow, inland EQs. The fault responsible for this EQ is considered to be one in the EnriquilloPlantain Garden fault system. Two significant aftershocks took place: the first one occurred at 17:00 on 12 January (LT) just after the main shock and another at 06:03 LT on 20 January (about eight days after the main shock). Both aftershocks had a magnitude of 5.9. There were reports of many casualties caused by this
EQ that 220 thousand people died and 320 thousand people were injured.

First of all, we have to indicate the relative location of the EQ epicenter and our VLF propagation path. A VLF/LF network so-called SAVNET has been established in South America (Raulin et al., 2009), and a few possible combinations of the VLF transmitter and receiving station are available for this Haiti EQ. Figure 19 illustrates the VLF transmitter, NAA (frequency $f=24.0 \mathrm{kHz}$ ) located in the east coast of USA (Cutler, geographic coordinates: $44.39^{\circ} \mathrm{N}, 76.12^{\circ} \mathrm{W}$ ) and our $\mathrm{VLF}$ receiving station of PLO (Punta Lobos, $12.50^{\circ} \mathrm{S}$, $76.80^{\circ} \mathrm{W}$, about $60 \mathrm{~km}$ south of Lima) in Peru. The great-circle path of this transmitter-receiver combination and also its corresponding fifth Fresnel zone are plotted in Figure 19. The EQ epicenter is found to be located exactly within the wave sensitive area, and we would expect significant EQ signatures within the VLF data set. The VLF/LF receiving system as described in Raulin et al. (2009) is quite similar to that used for the Japanese network in subsection 3.2, and the data sampling is 1 second.

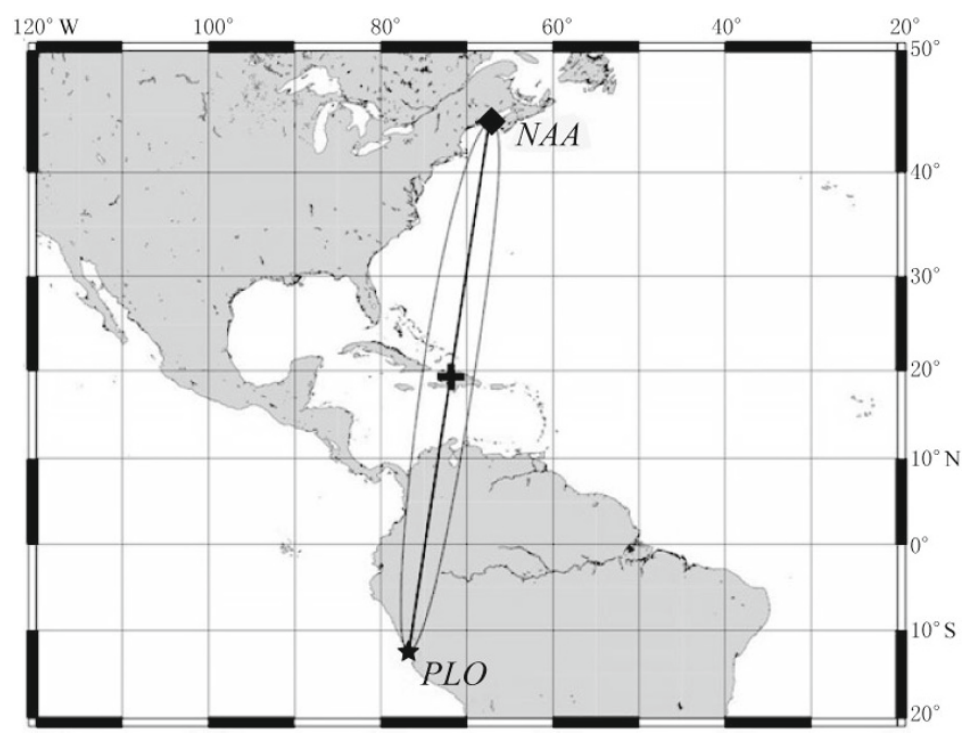

Figure 19 Relative location of the VLF transmitter (NAA), a VLF receiving station in Peru (PLO) and the EQ epicenter (indicated by a cross). The great circle path and the fifth Fresnel zone are also indicated.

We again use the nighttime fluctuation method for the analysis of this Haiti EQ. The propagation distance from the transmitter, NAA, to our VLF/LF station of PLO is $6.46 \mathrm{Mm}$, which is found to be comparable to that used in Kasahara et al. (2010). First we choose the nighttime period between $\mathrm{UTC}=0-6 \mathrm{~h}(\mathrm{LT}=\mathrm{UTC}-3$ h) as local nighttime for this propagation path. Same as before, we estimate the following three physical pa- rameters: (1) trend, (2) dispersion, and (3) NF. These quantities are normalized by their corresponding standard deviations.

Figure 20 depicts the obtained temporal evolutions of these three physical parameters (from top, trend, dispersion and NF). Grey parts indicate periods of lack of observation due to maintenance or failure in the system. We have analyzed the data from the beginning of 

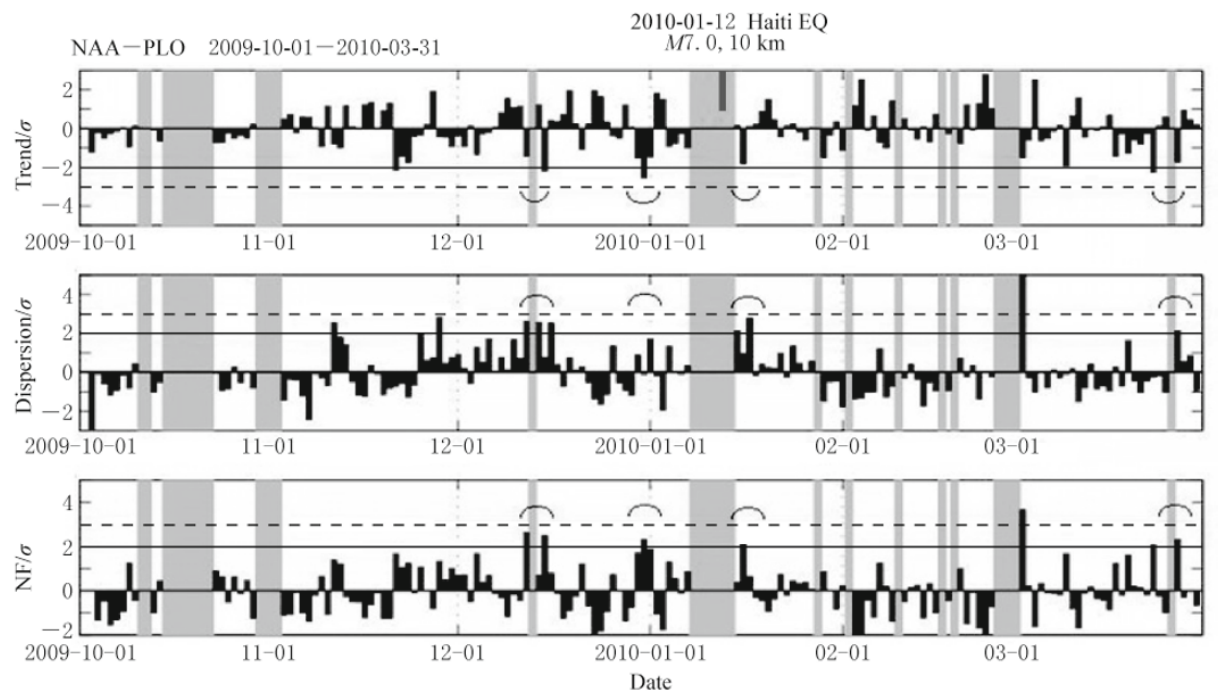

Figure 20 Temporal evolutions of three physical parameters of VLF propagation characteristics (from the top, trend, dispersion and NF). All of these parameters are normalized with the corresponding standard deviation $(\sigma)$ before the current day ( -30 to -1 day of the current day). Grey areas indicate no observation. The date is from the beginning of October, 2009 to the end of March, 2010, and EQ occurrence day is approximately located in the middle of our period. Of particular interest are the periods when the trend is decreased and the dispersion and NF show nearly simultaneous increases.

October, 2009 to the beginning of April, 2010 (seven months) as a preliminary analysis. Instead of looking at the temporal variations of each physical parameter separately, it is more appropriate to look at them globally. Our previous work by Kasahara et al. (2010) for medium-distance propagation paths (nearly the same distance as in the present Haiti case) as shown in section 4.2 indicates that the VLF/LF anomaly is characterized by a significant decrease in trend along with simultaneous increases in dispersion and NF, as it was found for short-distance propagation cases (Hayakawa et al., 2010b). Therefore, it is better to pay attention to the global variations of the three parameters as a pattern. When we look at Figure 20 in such a way, we can notice a few time periods, marked using arcs, during which the trend as the most important parameter is depleted exceeding its $2 \sigma$ ( $\sigma$ : standard deviation) criterion, and both dispersion and NF are enhanced by exceeding or approaching their corresponding $2 \sigma$ criteria. It is not important to have these properties exactly on the same day, but during the same period of a few days. From the periods satisfying this criterion, the first one corresponds to the middle of December, 2009 and the second is just around the New Year's day of 2010. The third one is just after the main shock. The last one is nearly at the end of our analysis period at the end of
March, 2010, which will be discussed later. The second period just around New Year's day is likely considered to be a clear precursor to the 2010 Haiti EQ, because the time lag of about 12 days is very consistent with the result by both previous case studies (e.g., Hayakawa, 2009a, 2010) and statistical studies (Hayakawa et al., 2010b). As for this period, the most important quantity, the trend, appears to be very much depleted exceeding $2 \sigma$ level. The second parameter, dispersion is seen to increase and approach the $2 \sigma$ line and the third parameter of NF is found to be enhanced exceeding the $2 \sigma$ criterion. Therefore, this anomaly is highly likely to be a precursor to the Haiti EQ. A few days just after the main shock of 12 January, 2010 (this is the third period), we observe another anomaly, in which we find a decrease in trend (close to but not exceeding the $2 \sigma$ line) and simultaneous increases in dispersion and NF. This might be an after-effect of the main shock.

We then pay attention to the additional periods of anomalous VLF/LF behaviors. One conspicuous anomaly around 12 December, 2009, about one month before the main shock, is characterized by the simultaneous decrease in trend and increases in dispersion and NF, so that it is reasonable to regard this as being seismogenic, though we do not know in which way this anomaly is related to the Haiti EQ. Of course, it seems 
unrealistic to say that this anomaly is the conventional precursor to the main shock because Hayakawa et al. (2010b) have indicated that the precursor appears one week to 10 days before an EQ. An additional, but less conspicuous, peak can be seen just around 12 November, 2009, for which we see a certain decrease in trend and the simultaneous significant increases in dispersion and NF, again suggesting a seismogenic effect. We have to think of a possibility to consider these two anomalous time periods with seismogenic property in a way consistent with the main shock of the Haiti EQ. One possible way might be the effect of Earth's tides as in Tanaka et al. (2004), who have shown a statistical evidence on the tidal effect in EQ occurrence (the synodic month of 29.5 days). The temporal spacing among 12 November, around 12 December and the main shock on 12 January, is found to be approximately one month, which might then be attributed to effect of Earth's tides. This kind of Earth's tides can be seen only in association with huge EQs (Tanaka, 2006), and in this sense the present 2010 Haiti EQ is such an example.

Finally, we discuss the last VLF anomaly on around 20 March, 2010. Of course, this anomaly is not associated with any of the aftershocks of the Haiti EQ, so that we have looked at any other EQs taking place within or around the wave sensitive area. We can list the following three EQs: 3 January $(M=5.7, d$ (depth) $=140$ $\left.\mathrm{km} ; 9.03^{\circ} \mathrm{S}, 78.05^{\circ} \mathrm{W}\right), 25$ January $(M=5.8, d=154 \mathrm{~km}$; $\left.8.47^{\circ} \mathrm{S}, 74.73^{\circ} \mathrm{W}\right)$ and 28 March $(M=5.3, d=56 \mathrm{~km}$; $\left.10.57^{\circ} \mathrm{S}, 78.81^{\circ} \mathrm{W}\right)$. The first two EQs are too deep to induce any ionospheric perturbations as based on the previous results (Kasahara et al., 2010), but the last and shallow EQ on 28 March is a possible candidate to have induced a seismo-ionospheric perturbation, about eight days before the main shock. So, we can say that the last VLF anomaly around 20 March is likely to be a precursor of the EQ on 28 March.

The analysis of subionospheric propagation from the transmitter of NAA $(24 \mathrm{kHz})$ to a VLF receiving station at PLO in Peru has yielded the following findings for the 2010 Haiti EQ.

1) The nighttime fluctuation method is found to be effective for a medium distance (6.43 Mm) VLF propagation path.

2) The VLF anomaly characterized by a significant decrease in trend and simultaneous enhancements of dispersion and NF, is found to be detected about 12 days before the main shock of the 2010 Haiti EQ.

3) Additional anomalies are observed around 12 November and 12 December, 2009, which are likely to be a signature of Earth's tides in the data set used for the Haiti EQ.

As for point (1), the effectiveness of the nighttime fluctuation method to this Haiti EQ case is considered to be a further confirmation of our recent paper by Kasahara et al. (2010) and presented in subsection 4.2. Then the propagation anomaly for this Haiti EQ is characterized by the significant decrease in trend, together with the simultaneous enhancements both in dispersion and NF (point (2)), which seems to be consistent with the conclusion obtained by Kasahara et al. (2010). The behavior of the VLF anomaly described in this paper, is exactly the same as that found for short-distance (less than 2-3 Mm) propagations (Hayakawa et al., 2010b). The epicenter of the present EQ happened to be located approximately on the great-circle path well within the wave sensitive area, so that it is very reasonable and acceptable for us to anticipate such a clear VLF propagation anomaly. The lead time of the anomaly found for this Haiti case is about 12 days, which seems to be slightly larger than the usual value of one week reported in Hayakawa et al. (2010b), but is not an unfavorable value. As it was mentioned as point (3), additional VLF propagation anomalies seem to be observed one month and two months before the main shock of the EQ. The effect of Earth's tides was recently found to be also observed in seismo-electromagnetic phenomena as in Hayakawa et al. (2009). This 2010 Haiti EQ was large enough with magnitude of 7.0 such as to be associated with the effect of Earth's tide. Finally, an additional VLF anomaly has been found in association to a subsequent shallow EQ in the northern part of Peru about eight days later.

This is a preliminary analysis result, and many things are still to be done. By using the network observation for different combinations of transmitters and receivers, we will analyze, in details, the temporal changes and spatial scale and structure of the ionospheric perturbation associated with this 2010 Haiti EQ, which would provide much more information on the lithosphere-ionospheric coupling mechanism.

4.6 Mechanism of seismo-ionospheric perturbation and lithosphere-atmosphereionosphere coupling

As extensively confirmed by means of statistics and case studies and the study on the modulation effects, etc., it seems highly likely that the ionosphere is disturbed before an EQ. But it is poorly understood how the ionosphere is perturbed by the precursory seismic activity in the lithosphere. Hayakawa et al. 
(2004a, b) have already proposed a few possible hypotheses on the mechanism of coupling between the lithospheric activity and ionosphere: (1) chemical channel, (2) AW and AGW channel, and (3) electromagnetic channel. Figure 21 illustrates the schematic diagram of these three coupling channels. As for the first channel, radon emanation induces the perturbation in the conductivity of the atmosphere, the change in the atmospheric electric field, then leading to the ionospheric modification through the atmospheric electric field (e.g., Miyaki et al., 2002; Pulinets and Boyarchuk, 2004; Sorokin et al., 2006). The second channel is based on the key role of atmospheric oscillations (AW or AGW) in the lithosphere-atmosphere-ionosphere coupling, and the perturbation in the Earth's surface (such as temperature, pressure) in a seismo-active region excites the atmospheric oscillations traveling up to the ionosphere and inducing the ionospheric density perturbations (Molchanov et al., 2001; Shvets et al., 2004; Korepanov et al., 2009). The last mechanism of electromagnetic channel is that the radio emissions (in any frequency range) generated in the lithosphere propagate up to the ionosphere, and modify the ionosphere thereby heating and/or ionization. But this mechanism is found to be insufficient because of the weak intensity of lithospheric radio emissions (Molchanov et al., 1993). So, the first and second mechanisms are likely plausible candidates for this coupling at the moment (Molchanov and Hayakawa, 2008). Pulinets and Boyarchuk (2004) insisted the first chemical channel as the most promising candidate for the ionospheric perturbations associated with EQs. That is, the emanation of radon is suggested as an important agent of seismo-ionospheric perturbation, but there seems to be very few experimental (observational) evidence in support of their hypothesis. Of course, we know that there have been reported on the radon emanation itself (e.g., Molchanov and Hayakawa, 2008) as a precursor to an EQ. But, it is poorly understood whether the radon emanation might result in the ionospheric perturbation. If so, how it is realized, is not well understood, though there have been very few papers on the correlation between the Earth's surface information (such as surface latent heat flux) and ionospheric perturbation as seen from VLF/LF subionospheric perturbation (Cervone et al., 2006).

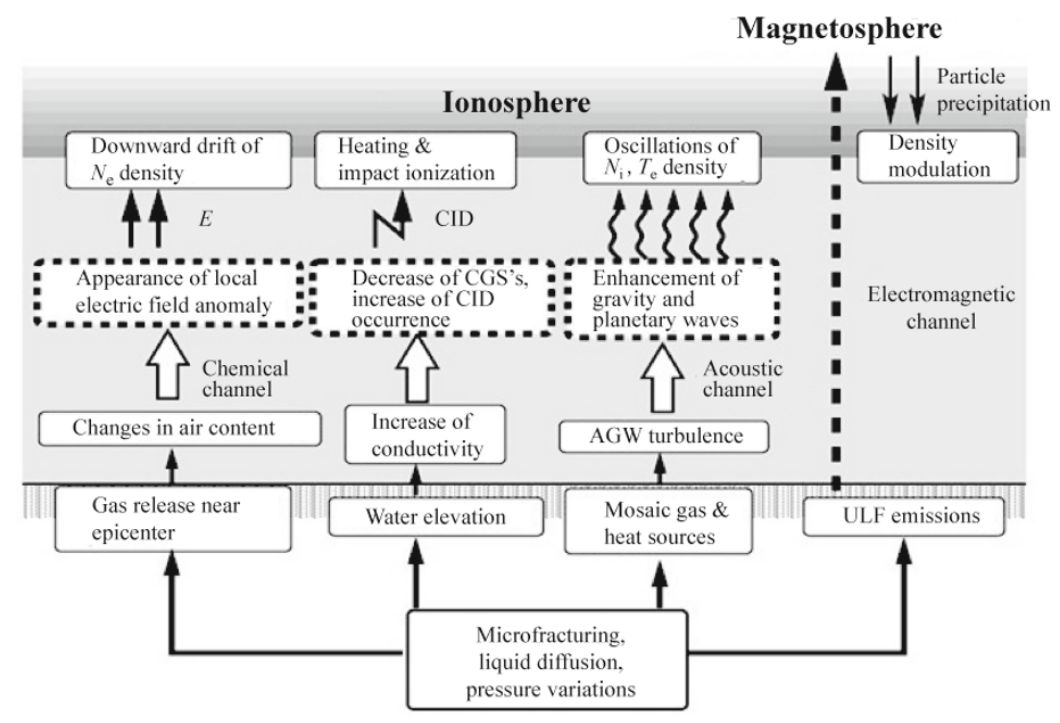

Figure 21 Schematic illustration of the lithosphere-atmosphere-ionosphere coupling and three channels, (1) chemical (+electric field) channel, (2) AW and AGW channel, and (3) electromagnetic channel. After Hayakawa (2004, 2009a, 2010) and Hayakawa et al. (2004a, b).

As compared with the first chemical channel, there have been accumulated a lot of indirect evidence on the importance of the second channel (due to atmospheric oscillations) mainly by using the VLF/LF subionospheric data. The results based on those subionospheric $\mathrm{VLF} / \mathrm{LF}$ data as presented in this paper are definitely providing us with the information taking place in the ionosphere, but the information is not so direct effects. So it is highly required to obtain any evidence on the direct coupling for this channel. For example, Korepanov et al. (2009) have made the first attempt in this direction by making full use of the data of ground-based 
pressure and magnetic field and satellite-based plasma.

Further studies are extensively required before we come to the conclusion on which channel is more relevant. Even though it is clear that the second channel is more probable at the moment, we need to carry out further works before a definite conclusion. Some works on the first channel are required as well.

\section{World-wide VLF/LF network observation}

\subsection{Japanese network}

As mentioned in this review, we have published a lot of papers on the basis of long-term observations by means of our established Japanese VLF/LF network. As our further extension of our Japanese network, an additional station at Kamchatka (KCK) was already established several years ago, and it is working perfectly, leading to a lot of interesting papers (Rozhnoi et al., 2004, 2007). As a further extension, we have established one station in Taiwan region of China (Hayakawa et al., 2010a), which suggested that this would be of extreme importance in finding out EQs in Taiwan and Okinawa areas.

\subsection{European VLF / LF network}

In collaboration with the group at University of Bari, Italy led by Prof. P. F. Biagi, we established one station in Bari by installing our VLF/LF system many years ago. By receiving several transmitters located within Europe even at one station, his group has already published a lot of interesting papers on the seismo-ionospheric perturbations associated with EQs in Europe (Biagi et al., 2004, 2007, 2009).

Prof. Biagi made a proposal of establishing a European VLF/LF network in the EGU meeting in 2008, and scientists from many countries showed an interest in participating in this project, including Austria, Greece, Romania, Turkey, and so on. Unfortunately in this proposal they intend to monitor only the amplitude from any VLF/LF transmitter. The homepage of this network is found at the site http://beta. *iscal.uniba.it/infrep/. The observation has already been started since October of 2009, and we hope a lot of significant results would be obtained from this network. The advantage of this European network is that EQs are not frequent in Europe, so that it will be very easy to find the one-to-one correspondence between an anomaly in subionospheric VLF/LF propagation and an EQ, unlike the situation in Japan.

\subsection{Indian network}

The group at Agra, India led by Prof. B. Singh has been continuing the measurement of subionospheric VLF/LF signals by using the similar equipment as used in Japan. They have published several papers on different subjects by using their VLF/LF data (Singh et al., 2004; Singh et al., 2005), including the seismic effect and also lightning effect (Trimpis) onto the ionosphere. The VLF/LF observation based on the reception of an Indian transmitter and foreign transmitters including NWC is still going on at Agra.

Also, I have found that the group led by Prof. S. Chakrabarti at Kolkata has recently organized an excellent VLF/LF network within India, and they are going to extend it further. Based on this VLF/LF network, they have obtained some significant effect of EQs from their observation with the use of an Indian transmitter (VTX, $18.2 \mathrm{kHz}$ ) (Sasmal and Chakrabarti, 2009; Chakrabarti et al., 2009). Their interest includes not only the seismic effects, but also the solar flare effect, the effect of solar eclipse and exotic gamma-ray bursts, etc.

\subsection{Brazilian network}

A few years ago an extensive network of receiving subionospheric VLF/LF signals was established in Brazil and its neighboring countries (Peru, Chile, etc.), which is named the South America VLF NET work (SAVNET) (Raulin et al., 2009). The VLF/LF system at each station is similar to that used in the Japanese network, but the sampling frequency of their observation is set to 1 second. Their interest is initially the effect of solar flares onto the ionosphere (Pacini and Raulin, 2006; Raulin et al., 2006, 2010), but their interest is now extending to the extra-terrestrial effects such as the exotic gamma-ray bursts and the seismic effect as well because of frequent occurrence of relatively huge EQs in the south America. Our collaboration with the Brazilian colleagues has just resulted in the paper (Hayakawa et al., 2011) concerning the 2010 Haiti EQ.

\section{Conclusion}

There has been an increased interest in the use of subionospheric VLF/LF propagation during over a decade for the following two reasons. The first one is the finding of so-called classical trimpi effects due to the precipitation of high energy electrons into the lower ionosphere in terms of wave-particle interactions in the magnetosphere and the subsequent finding of early/fast trimpi effects due to the direct heating/ionosphere by 
a lightning discharge and related phenomena such as mesospheric optical emission phenomena (sprites etc.) (Rodger, 1999). The second important finding is the effect of EQs onto the ionosphere because a clear evidence was obtained for the Kobe EQ with the use of VLF Omega signal (Hayakawa et al., 1996b). Later a lot of works on seismo-ionospheric perturbations have followed, and it is recently recognized that the upper ionosphere is also definitely perturbed (Liu et al., 2006).

The author is very happy to understand that similar kinds of VLF/LF networks as established in Japan, are being organized in different regions of the globe. Finally, I would like to emphasize that the international collaboration is highly required in order to have a better observational network and to exchange the data and ideas in future.

\section{References}

Al'pert Ya L (1983). The Near Earth and Interplanetary Plasma. Cambridge University Press, Cambridge, 250pp.

Biagi P F, Castellana L, Maggipinto T, Maggipinto G, Minafra A, Ermini A, Capozzi V, Perna G, Solovieva M, Rozhnoi A, Molchanov O A and Hayakawa M (2007). Decrease in the electric intensity of VLF/LF radio signals and possible connections. Nat Hazards Earth Syst Sci 7: 423-430.

Biagi P F, Castellana L, Maggipinto T, Maggipinto G, Minafra A, Ermini A, Molchanov O A, Rozhnoi A, Solovieva $M$ and Hayakawa M (2009). Anomalies in VLF radio signals related to the seismicity during NovemberDecember 2004: A comparison of ground and satellite results. Phys Chem Earth, Parts A/B/C 34(6-7): 456463.

Biagi P F, Piccolo R, Castellana L, Maggipinto T, Ermini A, Martellucci S, Bellecci C, Perna G, Capozzi V, Molchanov O A, Hayakawa M and Ohta K (2004). VLFLF radio signals collected at Bari (South Italy): a preliminary analysis on signal anomalies associated with earthquakes. Nat Hazards Earth Syst Sci 4: 685-689.

Budden K G (1961). The Radio Waves in the Ionosphere. Cambridge University Press, Cambridge, 524pp.

Cervone G, Maekawa S, Singh R P, Hayakawa M, Kafatos M and Shvets A (2006). Surface latent heat flux and nighttime LF anomalies prior to the $M_{\mathrm{W}}=8.3$ Tokachi-Oki earthquake. Nat Hazards Earth Syst Sci 6: 109-114.

Chakrabarti S K, Sasmal S and Chakrabarti S (2009). Ionospheric anomaly due to seismic activities - Part 2: Evidence from D-layer preparation and disappearance times. Nat Hazards Earth Syst Sci 10: 1 751-1 757.

Clilverd M A, Rodger C J and Thomson N R (1999). Investigating seismo-ionospheric effects on a long subionospheric path. J Geophys Res 104(A12): 28 171-28 179.

Dowden R L, Adams C P D, Brundell J B and Dowden P
E (1994). Rapid onset, rapid decay (RORD), phase and amplitude perturbations of VLF subionospheric transmissions. J Atmos Solar-Terr Phys 56: 1 513-1 527.

Fraser-Smith A C, Bernardi A, McGill P R, Ladd M E, Helliwll R A and Villard O G Jr. (1990). Low-frequency magnetic field measurements near the epicenter of the $M_{\mathrm{S}}$ 7.1 Loma Prieta earthquake. Geophys Res Lett 17: $1465-1468$.

Fujiwara H, Kamogawa M, Ikeda M, Liu J Y, Sakata H, Chen Y I, Ofuruton H, Muramatsu S, Chuo Y J and Ohtsuki Y H (2004). Atmospheric anomalies observed during earthquake occurrences. Geophys Res Lett 31: L17110, doi:10.1029/2004GL019865.

Fukumoto Y, Hayakawa M and Yasuda H (2001). Investigation of over-horizon VHF radio signals associated with earthquakes. Nat Hazards Earth Syst Sci 1: 107-112.

Gokhberg M B, Gufeld I L, Rozhnoi A A, Marenko V F, Yampolsky V S and Ponomarev E A (1989). Study of seismic influence on the ionosphere by super long wave probing of the Earth-ionosphere waveguide. Phys Earth Planet Inter 57: 64-67.

Gotoh K, Akinaga Y, Hayakawa M and Hattori K (2002). Principal component analysis of ULF geomagnetic data for Izu islands earthquakes in July 2000. J Atmos Electr 22: $1-12$.

Gotoh K, Hayakawa M and Smirnova N (2003). Fractal analysis of the gromagnetic data obtained at Izu Peninsula, Japan in relation to the nearby earthquake swarm of June-August 2000. Nat Hazards Earth Syst Sci 3: 229236.

Gotoh K, Hayakawa M, Smirnova N A and Hattori K (2004). Fractal analysis of seismogenic ULF emissions. Phys Chem Earth 29: 419-424.

Gufeld I L, Rozhnoi A A, Tyumensev S N, Sherstuk S V and Yampolsky V S (1992). Radiowave disturbances in period to Rudber and Rachinsk earthquakes. Phys Solid Earth 28(3): 267-270.

Hayakawa M (2004). Electromagnetic phenomena associated with earthquakes: A frontier in terrestrial electromagnetic noise environment. Recent Res Devel Geophysics 6: 81-112.

Hayakawa M (2007). VLF/LF radio sounding of ionospheric perturbations associated with earthquakes. Sensors 7: $1141-1158$.

Hayakawa M (2009a). Lower ionospheric perturbations associated with earthquakes, as detected by subionospheric $\mathrm{VLF} / \mathrm{LF}$ radio waves. In: Hayakawa $\mathrm{M}$ ed. Electromagnetic Phenomena Associated with Earthquakes. Transworld Research Network, Trivandrum (India), chapter 6, 137-185.

Hayakawa M ed. (2009b). Electromagnetic Phenomena Associated With Earthquakes. Transworld Research Network, Trivandrum (India), 279pp.

Hayakawa M (2009c). Seismogenic perturbation in the atmo- 
sphere. In: Hayakawa M ed. Electromagnetic Phenomena Associated with Earthquakes. Transworld Research Network, Trivandrum (India), chapter 5, 119-136.

Hayakawa M (2010). The use of subionospheric VLF/LF propagation for the study of lower ionospheric perturbations associated with earthquakes. In: Chakrabarti S K ed. Propagation Effects of Very Low Frequency Radio Waves. AIP Conf. Proc., 1286: 223-269.

Hayakawa M and Ida Y (2008). Fractal (mono- and multi-) analysis for the ULF data during the 1993 Guam earthquake for the study of prefracture criticality. Current Development in Theory and Applications of Wavelets 2(2): 159-174.

Hayakawa M and Molchanov O A (2007). Seismoelectromagnetics as a new field of radiophysics: Electromagnetic phenomena associated with earthquakes. Radio Science Bull 320: 8-17.

Hayakawa M and Molchanov O A eds. (2002). SeismoElectromagnetics: Lithosphere-Atmosphere-Ionosphere Coupling. TERRAPUB, Tokyo, 477pp.

Hayakawa M, Hattori K and Ohta K (2007a). Monitoring of ULF (ultra-low-frequency) geomagnetic variations associated with earthquakes. Sensors 7: 1 108-1 122.

Hayakawa M, Horie T, Muto F, Kasahara Y, Ohta K, Liu J Y and Hobara Y (2010a). Subionospheric VLF/LF probing of ionospheric perturbations associated with earthquakes: A possibility of earthquake prediction. SICE J Control, Measurement, and System Integration (SICE JCMSI) 3: 10-14.

Hayakawa M, Itoh T and Smirnova N (1999). Fractal analysis of ULF geomagnetic data associated with the Guam earthquake on August 8, 1993. Geophys Res Lett 26: 2 797-2 800.

Hayakawa M, Kasahara Y, Nakamura T, Muto F, Horie T, Maekawa S, Hobara Y, Rozhnoi A A, Solovieva M and Molchanov O A (2010b). A statistical study on the correlation between lower ionospheric perturbations as seen by subionospheric VLF/LF propagation and earthquakes. J Geophys Res 115: A09305, doi:10.1029/2009JA015143.

Hayakawa M, Kawate R, Molchanov O A and Yumoto K (1996a). Results of ultra-low-frequency magnetic field measurements during the Guam earthquake of 8 August 1993. Geophys Res Lett 23: 241-244.

Hayakawa M, Molchanov O A and NASDA/UEC team (2004a). Achievements of NASDA's Earthquake Remote Sensing Frontier Project. Terr Atmos Ocean Sci 15: 311-328.

Hayakawa M, Molchanov O A and NASDA/UEC team (2004b). Summary report of NASDA's earthquake remote sensing frontier project. Phys Chem Earth 29: 617625.

Hayakawa M, Molchanov O A, Ondoh T and Kawai E (1996b). The precursory signature effect of the Kobe earthquake on VLF subionospheric signals. J Comm Res Lab 43: 169-180.

Hayakawa M, Raulin J P, Kasahara Y, Bertoni F C P, Hobara Y and Guevara-Day W (2011). Ionospheric perturbations in possible association with the 2010 Haiti earthquake, as based on medium-distance subionospheric VLF propagation data. Nat Hazards Earth Syst Sci 11: 513518.

Hayakawa M, Sue Y and Nakamura T (2009). The effect of earth tides as observed in seismo-electromagnetic precursory signals. Nat Hazards Earth Syst Sci 9: 1 733-1 741.

Hayakawa M, Surkov V V, Fukumoto Y and Yonaiguchi N (2007b). Characteristics of VHF over-horizon signals possibly related to impending earthquakes and a mechanism of seismo-atmospheric perturbations. J Atmos Solar-Terr Phys 69: 1 057-1 062.

Horie T, Maekawa S, Yamauchi T and Hayakawa M (2007). A possible effect of ionospheric perturbations associated with the Sumatra earthquake, as revealed from subionospheric very-low-frequency (VLF) propagation (NWCJapan). Int'l J Remote Sensing 28(13): 3 133-3 139.

Ida Y and Hayakawa M (2006). Fractal analysis for the ULF data during the 1993 Guam earthquake to study prefracture criticality. Nonlinear Processes Geophys 13: 409-412.

Ida Y, Hayakawa M, Adalev A and Gotoh K (2005). Multifractal analysis for the ULF geomagnetic data during the 1993 Guam earthquake. Nonlinear Processes Geophys 12: $157-162$.

Imamura T, Ida Y, Kasahara Y, Nakamura T, Hobara Y and Hayakawa M (2010). Fractal analysis of subionospheric LF propagation data and consideration of the lithosphere-atmosphere-ionosphere coupling. Nat Hazards Earth Syst Sci 10: 901-906.

Inan V S, Pasko V and Bell T F (1996). Sustained heating of the ionosphere above thunderstorms as evidenced in "early/fast" events. Geophys Res Lett 23: $1067-1070$.

Kasahara Y, Muto F, Hobara Y and Hayakawa M (2010). The ionospheric perturbations associated with Asian earthquakes as seen from the subionospheric propagtion from NWC to Japanese stations. Nat Hazards Earth Syst Sci 10: 581-588.

Kasahara Y, Muto F, Horie T, Yoshida M, Hayakawa M, Ohta K, Rozhnoi A, Solovieva M and Molchanov O A (2008). On the statistical correlation between the ionospheric perturbations as detected by subionospheric VLF/LF propagation anomalies and earthquakes. Nat Hazards Earth Syst Sci 8: 653-656.

Kikuchi T and Evans D S (1983). Quantitative study of substorm-associated VLF phase anomalies and precipitating energetic electrons on November 13, 1979. J Geophys Res 88: 871-880.

Kleimenova N G, Kozyreva O V, Rozhnoi A A and Solovieva M S (2004). Variations in the VLF signal parameters 
on the Australia-Kamchatka radio path during magnetic storms. Geomag Aeron 44: 385-393.

Kopytenko Y, Matiashvili T G, Veronov P M and Molchanov O A (1993). Detection of ultra-low frequency emissions connected with the Spitak earthquake and its aftershock activity, based on geomagnetic pulsation data at Dusheti and Vardzia observatories. Phys Earth Planet Inter 77: 85-95.

Korepanov V, Hayakawa M, Yampolski Y and Lizunov G (2009). AGW as a seismo-ionospheric coupling responsible agent. Phys Chem Earth, Parts $A / B / C$ 34(6-7): 485-495.

Liu J Y, Chen Y I and Chuo Y J (2006). A statistical investigation of pre-earthquake ionospheric anomaly. J Geophys Res 111: A05304, 10.1029/2005JA011333.

Maekawa S and Hayakawa M (2006). A statistical study on the dependence of characteristics of VLF/LF terminator. IEEJ Trans Fundamentals and Materials 126(4): 220226 .

Maekawa S, Horie T, Yamauchi T, Sawaya T, Ishikawa M, Hayakawa M and Sasaki H (2006). A statistical study on the effect of earthquakes on the ionosphere, based on the subionospheric LF propagation data in Japan. Ann Geophysicae 24: 2 219-2 225.

Mitra A P (1974). Ionospheric Effects of Solar Flares. Springer, New York, 320pp.

Miyaki K, Hayakawa M and Molchanov O A (2002). The role of gravity waves in the lithosphere-ionosphere coupling, as revealed from the subionospheric LF propagation data. In: Hayakawa $\mathrm{M}$ and Molchanov O A eds. Seismo Electromagnetics: Lithosphere-AtmosphereIonosphere Coupling. TERRAPUB, Tokyo, 229-232.

Molchanov O A and Hayakawa M (1995). Generation of ULF electromagnetic emissions by microfracturing. Geophys Res Lett 22: 3 091-3 094.

Molchanov O A and Hayakawa M (1998). Subionospheric VLF signal perturbations possibly related to earthquakes. J Geophys Res 103: 17 489-17 504.

Molchanov O A and Hayakawa M (2008). SeismoElectromagnetics and Related Phenomena: History and Latest Results. TERRAPUB, Tokyo, 189pp.

Molchanov O A, Hayakawa M and Miyaki K (2001). VLF/LF sounding of the lower ionosphere to study the role of atmospheric oscillations in the lithosphereionosphere coupling. Adv Polar Upper Atmos Res 15: 146-158.

Molchanov O A, Hayakawa M, Ondoh T and Kawai E (1998). Precursory effects in the subionospheric VLF signals for the Kobe earthquake. Phys Earth Planet Inter 105: 239-248.

Molchanov O A, Mazhaeva O A, Goliavin A N and Hayakawa M (1993). Observations by the intercosmos-24 satellite of ELF-VLF electromagnetic emissions associated with earthquakes. Ann Geophys 11: 431-440.
Muto F, Horie T, Yoshida M, Hayakawa M, Rozhnoi A, Solovieva M and Molchanov O A (2009a). Ionospheric perturbations related to the Miyagi-oki earthquake on 16 August 2005, as seen from Japanese VLF/LF subionospheric propagation network. Phys Chem Earth, Parts $A / B / C$ 34(6-7): 449-455.

Muto F, Kasahara Y, Hobara Y, Hayakawa M, Rozhnoi A, Solovieva M and Molchanov O A (2009b). Further study on the role of atmospheric gravity waves on the seismoionospheric perturbations as detected by subionospheric VLF/LF propagation. Nat Hazards Earth Syst Sci 9: $1111-1118$.

Nagao T, Enomoto Y, Fujinawa Y, Hata M, Hayakawa M, Huang Q, Izutsu J, Kushida Y, Maeda K, Oike K, Uyeda S and Yoshino T (2002). Electromagnetic anomalies associated with 1995 Kobe earthquake. J Geodynamics 33: $477-487$.

Pacini A A and Raulin J P (2006). Solar X-ray flares and ionospheric sudden phase anomalies relationship: A solar cycle phase dependence. J Geophys Res 111: A09301, doi:10.1029/2006JA011613.

Pulinets S A and Boyarchuk K (2004). Ionospheric Precursors of Earthquakes. Springer, Berlin, 315pp.

Raulin J P, Bertoni F C P, Gawilan H R, Guevara-Day W, Rodriguez R, Fernandez G, Correia E, Kaufman P, Pacini A, Stekel T R C, Lima W L C, Schuch N J, Fagundes P R and Hadano R (2010). Solar flare detection sensitivity using the South America VLF Network (SAVNET). J Geophys Res 115: A07301, doi:10.1029/2009JA015154.

Raulin J P, David P, Hadano R, Saraiva A C V, Correia E and Kaufman P (2009). The South America VLF NETwork (SAVNET). Earth, Moon and Planets 104: 247261.

Raulin J P, Pacini A A, Kaufman P, Correia E and Martinez M A G (2006). On the detectability of solar X-ray flares using very low frequency sudden phase anomalies. $J$ Atmos Solar-Terr Phys 68: 1 029-1 035.

Rodger C and McCormic R J (2006). Remote sensing of the upper atmosphere by VLF. In: Füllekrug M, Mareev E A and Rycroft M J eds. Sprites, Elves and Intense Lighting Discharges. NATO Sci Series. Springer, Berlin, 167-190.

Rodger C J (1999). Red sprites, upward lightning, and VLF perturbations. Rev Geophys 37: 317-339.

Rozhnoi A, Molchanov O, Solovieva M, Gladyshev V, Akentieva O, Berthelier J J, Parrot M, Lefeuvre F, Hayakawa M, Castellana L and Biagi P F (2007). Possible seismoionosphere perturbations revealed by VLF signals collected on ground and on a satellite. Nat Hazards Earth Syst Sci 7: 617-624.

Rozhnoi R, Solovieva M S, Molchanov O A and Hayakawa M (2004). Middle latitude LF (40 kHz) phase variations associated with earthquakes for quiet and disturbed geomagnetic conditions. Phys Chem Earth 29: 589-598.

Sasmal S and Chakrabarti S K (2009). Ionospheric anoma- 
ly due to seismic activities - Part 1: Calibration of the VLF signal of VTX $18.2 \mathrm{kHz}$ station from Kolkata and deviation during seismic events. Nat Hazards Earth Syst Sci 9: 1 403-1 408.

Shvets A V, Hayakawa M and Molchanov O A (2002). Subionospheric VLF monitoring for earthquake-related ionospheric perturbations. J Atmos Electr 22: 87-99.

Shvets A V, Hayakawa M, Molchanov O A and Ando Y (2004). A study of ionospheric response to regional seismic activity by VLF radio sounding. Phys Chem Earth 29: $627-637$.

Singh B, Kushwah V, Singh V, Tomar M and Hayakawa M (2005). Simulataneous ULF/VLF amplitude anomalies observed during moderate earthquakes in India region. Indian J Radio Space Phys 34: 221-232.

Singh V, Singh B, Hayakawa M, Kumar M, Kushwah V and Singh O P (2004). Nighttime amplitude decrease in 19.8 $\mathrm{kHz}$ NWC signals observed at Agra possibly caused by moderate seismic activities along the propagation path. J Atmos Electr 24: 1-15.

Smirnova N, Hayakawa M and Gotoh K (2004). Precursory behavior of fractal characteristics of the ULF electromagnetic fields in seismic active zones before strong earthquakes. Phys Chem Earth 29: 445-451.

Smirnova N, Hayakawa M, Gotoh K and Volobuev D (2001). Scaling characteristics of ULF geomagnetic field at the Guam seismoactive area and their dynamics in relation to the earthquake. Nat Hazards Earth Syst Sci 1: 119126.

Sorokin V M, Yaschenko A K, Chmyrev V M and Hayakawa M (2006). DC electric field formation in the mid-latitude ionosphere over typhoon and earthquake regions. Phys Chem Earth 31: 454-461.

Sue Y (2009). The effect of earth tides in triggering earthquake as clearly observed in some specific regions of Japan. J Atmos Electr 29: 53-62.
Tanaka S (2006). Tidal triggering of earthquakes precursory to the $2004 M_{\mathrm{W}}=9.0$ off Sumatra earthquake. 4 th International Workshop on Statistical Seismology. Jan. 9-13, 2006, Kanagawa, Japan.

Tanaka S, Ohtake M and Sato H (2004). Tidal triggering of earthquakes in Japan related to the regional tectonic stress. Earth Planets Space 56: 511-515.

Tanaka Y, Terasawa T, Yoshida M, Horie T and Hayakawa M (2008). Ionospheric disturbances caused by SGR 1900+14 giant gamma ray flare in 1998: Constraints on the energy spectrum of the flare. J Geophys Res 113: A07307, doi:10.1029/2008JA013119.

Tronin A A, Hayakawa M and Molchanov O A (2002). Thermal IR satellite data application for earthquake research in Japan and China. J Geodynamics 33: 319-534.

Uyeda S, Hayakawa M, Nagao T, Molchanov O A, Hattori K, Orihara Y, Gotoh K, Akinaga Y and Tanaka H (2002). Electric and magnetic phenomena observed before the volcano-seismo activity in 2000 in the Izu Island region, Japan. Proc. US National Academy of Sci. (PNAS) 99: $7352-7355$.

Wait J R (1962). Electromagnetic Waves in Stratified Media. Pergamon Press, Oxford, 608pp.

Watt A D (1967). VLF Radio Engineering. Pergamon Press, Oxford, 703pp.

Yamauchi T, Maekawa S, Horie T, Hayakawa M and Soloviev O (2007). Subionospheric VLF/LF monitoring of ionospheric perturbations for the 2004 Mid-Niigata earthquake and their structure and dynamics. J Atmos SolarTerr Phys 69: 793-802.

Yoshida M, Yamauchi T, Horie T and Hayakawa M (2008). On the generation mechanism of terminator times in subionospheric VLF/LF propagation and its possible application to seismogenic effects. Nat Hazards Earth Syst Sci 8: 129-134. 